Y. SUZUKI

KODAI MATH. J.

19 (1996), $234-258$

\title{
CONSTRUCTION OF MARKOV PROCESSES OF CERTAIN SPIN SYSTEMS ON $Z$
}

\author{
YUKI SUZUKI
}

\section{Introduction}

In this paper we construct jump-type Markov processes of $\boldsymbol{R}$-valued spin fields on $\boldsymbol{Z}$. In such a process an $\boldsymbol{R}$-valued spin is attached to each site of $\boldsymbol{Z}$ and the values of spins evolve at random times. The values of spins on each pair of adjacent sites evolve simultaneously according to a certain law and the jump rate is a function of the values of spins. In the case where spins take values in a compact space, various particle systems have been constructed (cf. $[2])$. Spitzer ([3]) and Holley ([1]) have constructed particle systems in which spins take values in $\boldsymbol{R}$ and jump rates are bounded. Here we treat a case where the jump rate is unbounded. In such a case the construction of the process is not easy. In [6] we are going to discuss the hydrodynamic limit for a special class of our present models, namely, for a model which is reversible and of gradient type; owing to these properties the jump rate is specialized but what is emphasized is that it is unbounded. This is the reason we are interested in the case of unbounded jump rates.

The process we are going to construct has an invariant measure which is an infinite product of some measure on $\boldsymbol{R}$. We construct our process in equilibrium and in non-equilibrium, separately, assuming suitable conditions for an initial distribution. In the non-equilibrium case a rather strong entropy assumption for an initial distribution is required.

In the course of our construction, stochastic differential equations associated with Poisson random measures are used. The process we want to construct can be obtained as a limit of a sequence of finite spin systems. We also show that our process has a finite cluster property if the initial distribution, in addition to the assumption for the construction, is ergodic relative to the shift transformation on the configuration space.

This research was supported by the Japan Society for the Promotion of Science for Japanese Junior Scientists (April, 1993-March, 1995) and by Grant-in-Ald for Scientific Research (No. 015-10002667), Ministry of Education, Science and Culture.

Received April 26, 1995. 


\section{The model and main results}

Before introducing our model on $\boldsymbol{Z}$, we describe the dynamics for a system on two sites. Let $\pi(x, y ; d u d v)$ be a transition probability on $\boldsymbol{R}^{2}$ and $c(x, y)$ a non-negative Borel function on $\boldsymbol{R}^{2}$ which is locally bounded. Throughout this paper we assume that there exists a probability measure $\nu$ on $\boldsymbol{R}$ satisfying

$$
\iint \mathcal{\nu}(d x) \nu(d y) c(x, y) \pi(x, y ; B)=\iint_{B} c(x, y) \nu(d x) \nu(d y) \text { for all } B \in \mathscr{B}\left(\boldsymbol{R}^{2}\right),
$$

where $\mathscr{B}\left(\boldsymbol{R}^{2}\right)$ denotes the Borel field on $\boldsymbol{R}^{2}$, and

$$
\iint c(x, y)^{2} \nu(d x) \nu(d y)<\infty .
$$

The measure $\nu$ is called a reference measure. Examples of $\pi(x, y ; \cdot)$ and $c(x, y)$ satisfying the above assumption will be given at the end of this section. Let $\boldsymbol{C}_{b}\left(\boldsymbol{R}^{2}\right)$ be the set of bounded continuous functions on $\boldsymbol{R}^{2}$, and define an operator $L$ on $\boldsymbol{C}_{b}\left(\boldsymbol{R}^{2}\right)$ by

$$
L \phi(x, y)=c(x, y)\left\{\iint \phi(u, v) \pi(x, y ; d u d v)-\phi(x, y)\right\}, \quad \phi \in \boldsymbol{C}_{b}\left(\boldsymbol{R}^{2}\right) .
$$

The Markov process on $\boldsymbol{R}^{2}$ describing our system on two sites is generated by $L$. The assumption (A.1) implies that this process has an invariant measure $\nu \otimes \nu$ (see Lemma 2.1 in Section 2).

Now we describe our system on $\boldsymbol{Z}$. Let

$$
\mathscr{X}=\boldsymbol{R}^{\boldsymbol{Z}}=\left\{\boldsymbol{x}=\left(x_{\imath} ; i \in \boldsymbol{Z}\right): x_{i} \in \boldsymbol{R} \text { for } \imath \in \boldsymbol{Z}\right\} .
$$

It is a Polish space endowed with the product topology. Here $x_{\imath}$ is viewed as a value of a spin on a site $\imath$ and $\boldsymbol{x}$ as a configuration of values of spins on $\boldsymbol{Z}$. The process we are going to construct is a Markov process on $\mathscr{X}$, in which the values of spins $x_{\imath}$ and $x_{\imath+1}$ on each pair of adjacent sites evolve simultaneously according to $\pi\left(x_{\imath}, x_{\imath+1} ; \cdot\right)$ at rate $c\left(x_{\imath}, x_{\imath+1}\right)$. Roughly speaking, it is a Markov process with generator

$$
\mathcal{L} \boldsymbol{\phi}(\boldsymbol{x})=\sum_{\imath \in \boldsymbol{Z}} L_{i} \phi(\boldsymbol{x}), \quad L_{i} \phi(\boldsymbol{x})=L \boldsymbol{\phi}_{\boldsymbol{x}, i}\left(x_{\imath}, x_{\imath+1}\right),
$$

where $\phi_{x, i}(x, y)=\left.\phi(x)\right|_{x_{i}=x, x_{\imath+1}=y}$ is a function of $(x, y)$ with $\left(x_{k}\right)_{k \neq \imath, \imath+1}$ fixed. In the case of bounded $c(x, y)$ our process can be constructed easily for any initial value (see Remark 3.2 in Section 3), so the construction in the case of unbounded $c(x, y)$ is the essential part of this paper.

In the equilibrium case under the assumption of the existence of $\nu$ satisfying (A.1) and (A.2) we construct our equilibrium process on $\mathscr{X}$ with initial (therefore marginal) distribution $\nu^{\otimes \infty}$. Let $\mathscr{P}(\mathscr{X})$ denote the set of probability measures on $\mathscr{X}$. For the discussion of the non-equilibrium case we need to introduce, for any non-negative integer $m$, the relative entropy of $\mu \in \mathscr{P}(\mathscr{X})$ in 
$\{-m, \cdots, m\}$ with respect to the product measure $\nu^{\otimes(2 m+1)}$ defined by

$$
H_{m}(\mu)= \begin{cases}\int \log \left\{\left(d \mu_{m}\right) /\left(d \nu^{\otimes(2 m+1)}\right)\right\} d \mu_{m}, & \text { if } \mu_{m} \ll \nu^{\otimes(2 m+1)}, \\ \infty, & \text { otherwise, }\end{cases}
$$

where $\mu_{m}$ denotes the projection of $\mu$ to $\boldsymbol{R}^{(-m, \cdots, m\}}$. In the non-equilibrium case we assume the following conditions (A.3), (A.4) and (A.5) for a reference measure $\nu$ and an initial distribution $\mu^{0} \in \mathscr{P}(\mathscr{X})$ :

(A.3) There exists $\beta \geqq 2$ such that $\iint e^{r c(x, y)^{\beta}} \nu(d x) \nu(d y)<\infty$ for some $\gamma>0$.

(A.4) There exist $C>0$ and $\alpha \geqq 0$ such that $H_{m}\left(\mu^{0}\right) \leqq C m^{\alpha}$ for all $m \geqq 1$.

(A.5) $\alpha$ in (A.4) and $\beta$ in (A.3) satisfy the relation

$$
\begin{cases}\beta>\left(2 \alpha+1+\sqrt{4 \alpha^{2}+1}\right) / 2, & \text { if } \alpha \geqq 2 / 3, \\ \beta=2, & \text { if } 0 \leqq \alpha<2 / 3 .\end{cases}
$$

Note that (A.3) implies (A.2). Examples of $\mu^{0} \in \mathscr{L}(\mathscr{X})$ satisfying (A.4) will be given in Section 3 (see Remark 3.1).

In order to construct our process, let us introduce finite systems. Let $M=$ $\left\{M^{-}, M^{+}\right\}$be a pair of integers $M^{-}$and $M^{+}$such that $M^{-} \leqq-1$ and $M^{+} \geqq 1$ and let

$$
\boldsymbol{X}^{M}(t)=\left(X_{\imath}^{M}(t) ; i \in \boldsymbol{Z}\right), \quad t \geqq 0,
$$

be the Markov process on $\mathscr{X}$ generated by $\mathcal{L}^{M}=\Sigma L_{\imath}$, where the summation is taken over the integers $i$ 's such that $M^{-} \leqq i<M^{+}$. By (A.1) and (A.2), this process is conservative for almost all initial values with respect to $\nu^{\otimes \infty}$ and has an invariant measure $\nu^{\otimes \infty}$ (see Lemma 2.1 in Section 2). In this process, only the values of spins on each pair of adjacent sites in $\left[\mathrm{M}^{-}, \mathrm{M}^{+}\right]$evolve simultaneously and the values of spins on sites outside $\left[M^{-}, M^{+}\right]$remain unchanged with time. We obtain our process as a limit of $X^{M}(t), t \geqq 0$, as $M^{-} \rightarrow-\infty$, $M^{+} \rightarrow+\infty$. To do this, we construct $\boldsymbol{X}^{M}(t), t \geqq 0$, on a common probability space for all $M^{-} \leqq-1$ and $M^{+} \geqq 1$ by solving certain stochastic differential equations (abbreviated to SDE's) associated with common Poisson random measures. Let $(\Omega, \widetilde{F}, \boldsymbol{P})$ be a probability space equipped with a suitable filtration $\left\{\mathfrak{F}_{t}\right\}_{t \geqq 0}$. Let $\left\{N_{i}(d s d \eta d \xi), i \in Z\right\}$ be an independent family of $\mathfrak{F}_{t}$-adapted Poisson random measures on $(0, \infty) \times(0, \infty) \times(0,1]$ with intensity measure $d s d \eta d \xi$, and let $\boldsymbol{X}=\left(X_{\imath} ; i \in \boldsymbol{Z}\right)$ be an $\mathscr{X}$-valued random variable which is distributed according to $\mu^{0} \in \mathscr{P}(\mathscr{X})$ and is $\widetilde{\mho}_{0}$-measurable (so is independent of $\left.\left\{N_{i}(\cdot), i \in Z\right\}\right)$. We choose Borel functions $a(\xi ; x, y)$ and $b(\xi ; x, y)$ defined on $(0,1] \times \boldsymbol{R}^{2}$ in such a way that, for fixed $x$ and $y,(a(\xi ; x, y), b(\xi ; x, y))$ is an $\boldsymbol{R}^{2}$-valued random variable on the probability space $((0,1], d \xi)$ having probability distribution $\pi(x, y ; \cdot)$. For each $M^{-} \leqq-1$ and $M^{+} \geqq 1$, we can construct a process $\boldsymbol{X}^{M}(t), t \geqq 0$, with initial distribution $\mu^{0}$ on $(\Omega, \mathfrak{F}, \boldsymbol{P})$ as the unique solution of the SDE 


$$
\begin{aligned}
X_{\imath}^{M}(t)= & X_{i}+\mathbf{1}_{\left[M^{-}, M^{+}\right)}(i) \int_{0}^{t} \int_{0}^{c\left(X_{\imath}^{M}(s-), X_{\imath+1}^{M}(s-)\right)} \int_{0}^{1} \\
& \left\{a\left(\xi ; X_{\imath}^{M}(s-), X_{\imath+1}^{M}(s-)\right)-X_{\imath}^{M}(s-)\right\} N_{i}(d s d \eta d \xi) \\
+ & \mathbf{1}_{\left[M^{-}, M^{+}\right)}(\imath-1) \int_{0}^{t} \int_{0}^{c\left(X_{\imath-1}^{M}(s-), X_{\imath}^{M}(s-)\right)} \int_{0}^{1} \\
& \left\{b\left(\xi ; X_{\imath-1}^{M}(s-), X_{\imath}^{M}(s-)\right)-X_{\imath}^{M}(s-)\right\} N_{\imath-1}(d s d \eta d \xi), \quad i \in Z, t \geqq 0 .
\end{aligned}
$$

Here $\mathbf{1}_{A}$ stands for the indicator function of a set $A$. The construction of $\boldsymbol{X}^{M}(t), t \geqq 0$, that is, solving the $\operatorname{SDE}(1.3)$ will be described in Section 2 .

The main results of this paper are the following.

THEOREM 1.1. Suppose that there is a reference measure $\nu$ satisfying (A.1) and (A.2) and let $\mu^{0}=\nu^{\otimes \infty}$. Let $M_{k}=\left\{M_{k}^{-}, M_{k}^{+}\right\}, k \geqq 1$, satisfy $-1 \geqq M_{1}^{-} \geqq M_{2}^{-} \geqq$ $\cdots, 1 \leqq M_{1}^{+} \leqq M_{2}^{\perp} \leqq \cdots$ and

$$
\sum_{k=1}^{\infty}\left(\left|M_{k}^{-}\right| \wedge M_{k}^{+}\right)^{-1 / 2}<\infty
$$

Then for any $T>0$ we have

$$
\begin{aligned}
& \boldsymbol{P}\left\{\text { for any } i \in \boldsymbol{Z} \text { there exists } X_{i}(t) \text { such that } X_{i}(t)=X_{\imath}^{M_{k}}(t)\right. \\
& \quad \text { for all } t \in[0, T] \text { and for all sufficiently large } k\}=1 .
\end{aligned}
$$

Moreover $\boldsymbol{X}(t)=\left(X_{i}(t) ; i \in \boldsymbol{Z}\right), t \geqq 0$, depends neither on $\left\{M_{k}, k \geqq 1\right\}$ satisfying (A.6) nor on $T$, and is an equilibrium Markov process on $\mathscr{X}$ with invariant measure $\nu^{\otimes \infty}$ satisfying the $S D E$

$$
\begin{aligned}
& X_{i}(t)= X_{i}+\int_{0}^{t} \int_{0}^{c\left(X_{i}(s-), X_{\imath+1}(s-)\right)} \int_{0}^{1}\left\{a\left(\xi ; X_{i}(s-), X_{\imath+1}(s-)\right)-X_{i}(s-)\right\} \\
& N_{i}(d s d \eta d \xi) \\
&+\int_{0}^{t} \int_{0}^{c\left(X_{\imath-1}(s-), X_{i}(s-)\right)} \int_{0}^{1}\left\{b\left(\xi ; X_{\imath-1}(s-), X_{i}(s-)\right)-X_{i}(s-)\right\} \\
& N_{\imath-1}(d s d \eta d \xi), \quad i \in \boldsymbol{Z}, t \geqq 0 .
\end{aligned}
$$

THEOREM 1.2. Suppose that there is a reference measure $\nu$ satisfying (A.1) and (A.3) and that $\mu^{0}$ satisfies (A.4) and (A.5). Let $M_{k}=\left\{M_{k}^{-}, M_{k}^{+}\right\}, k \geqq 1$, satisfy $-1 \geqq M_{1}^{-} \geqq M_{2}^{-} \geqq \cdots, 1 \leqq M_{1}^{+} \leqq M_{2}^{+} \leqq \cdots$ and

(i) there exists $C>1$ such that $1 / C \leqq M_{k}^{+} /\left|M_{k}^{-}\right| \leqq C$ and $M_{k+1}^{+} \leqq C M_{k}^{+}$ for all sufficiently large $k$;

(ii) $\sum_{k=1}^{\infty}\left(M_{k}^{+}\right)^{-(q-1)}<\infty$, where $q=2+\alpha / \beta^{2}-(2 \alpha) / \beta-1 / \beta$ (which is strictly between 1 and 2 by virtue of (A.5)).

Then for any $T>0$ we have 


$$
\begin{aligned}
& \boldsymbol{P}\left\{\text { for any } i \in \boldsymbol{Z} \text { there exists } X_{i}(t) \text { such that } X_{i}(t)=X_{2}^{M}{ }_{k}(t)\right. \\
& \text { for all } t \in[0, T] \text { and for all sufficiently large } k\}=1 .
\end{aligned}
$$

Moreover $\boldsymbol{X}(t)=\left(X_{i}(t) ; i \in \boldsymbol{Z}\right), t \geqq 0$, depends neither on $\left\{M_{k}, k \geqq 1\right\}$ satisfying (A.7) nor on $T$, and is a Markov process on $\mathscr{X}$ with initıal distribution $\mu^{0}$ satisfying the $S D E$ (1.5).

For any $T>0$, let $\boldsymbol{D}[0, T]=\boldsymbol{D}([0, T] ; \mathscr{X})$ be the set of functions on $[0, T]$ with values in $\mathscr{X}$ which are right continuous and have left limits. For a generic element $\boldsymbol{x}(\cdot)$ of $\boldsymbol{D}[0, T]$, the value $(\in \mathscr{X})$ of $\boldsymbol{x}(\cdot)$ at time $t$ is denoted by $\boldsymbol{x}(t)$. Let $\mathcal{G}_{t}, t \geqq 0$, be the smallest $\boldsymbol{\sigma}$-field on $\boldsymbol{D}[0, T]$ with respect to which all $\boldsymbol{x}(s), 0 \leqq s \leqq t$, are measurable, and put $\mathcal{G}=\vee_{t} \mathcal{G}_{t}$. The process $\boldsymbol{X}(t), 0 \leqq t \leqq T$, obtained in Theorem 1.1 or Theorem 1.2 defines a random variable in $\boldsymbol{D}[0, T]$. Denote by $P_{\mu 0}$ the distribution of $\boldsymbol{X}(t), 0 \leqq t \leqq T$, when its initial distribution is $\mu^{0}$. This is a probability measure on $(\boldsymbol{D}[0, T], \mathcal{G})$. Denote by $\mathscr{D}$ the set of bounded Borel functions on $\mathscr{X}$ which depend only on finitely many coordinates. For $\phi \in \mathscr{D}, \mathcal{L} \phi$ in (1.1) is well-defined. By Itô's formula, we have for $\phi \in \mathscr{D}$,

$$
\begin{aligned}
\phi(\boldsymbol{X}(t))-\boldsymbol{\phi}(\boldsymbol{X})= & \sum_{\imath \in \boldsymbol{Z}} \int_{0}^{t} \int_{0}^{c\left(X_{i}(s-), X_{\imath+1}(s-)\right)} \int_{0}^{1} \\
& \left\{\phi_{\boldsymbol{X}(s-), i}\left(a\left(\xi ; X_{i}(s-), X_{\imath+1}(s-)\right), b\left(\xi ; X_{i}(s-), X_{\imath+1}(s-)\right)\right)\right. \\
& -\phi(X(s-))\} N_{i}(d s d \eta d \xi) .
\end{aligned}
$$

Therefore $\phi(\boldsymbol{x}(t))-\boldsymbol{\phi}(\boldsymbol{x}(0))-\int_{0}^{t} \mathcal{L} \phi(\boldsymbol{x}(s)) d s$ is a $\left\{G_{t}\right\}$-martingale under $P_{\mu 0}$ for all $\phi \in \mathscr{D}$. In this sense $\left\{\boldsymbol{x}(t), 0 \leqq t \leqq T, P_{\mu 0}\right\}$ is associated with $\mathcal{L}$ and may be regarded as a Markov process describing our model.

Now we discuss a finite cluster property of $\left\{\boldsymbol{x}(t), 0 \leqq t \leqq T, P_{; 0}\right\}$. For the sake of convenience we say that the $i$-th and the $(i+1)$-th spins of the process $\{\boldsymbol{X}(\cdot)\}$ interact with each other (or simply, (i, $i+1)$-interaction takes place) at time $t$ if

$$
\int_{0}^{t} \int_{0}^{c\left(X_{i}(s-), x_{\imath+1}(s-)\right)} \int_{0}^{1} N_{i}(d s d \eta d \xi)>\int_{0}^{t-} \int_{0}^{c\left(x_{i}(s-), x_{\imath+1}(s-)\right)} \int_{0}^{1} N_{i}(d s d \eta d \xi) .
$$

The finite cluster property we are going to formulate says roughly that, with probability 1 , there are infinitely many $i$ 's such that $(i, i+1)$-interaction does not take place up to time $T, T>0$ being an arbitrarily given constant.

Before describing our result concerning a finite cluster property, we introduce some notation. First we introduce a shift transformation $\Theta$ on $\mathscr{X}$. For $\boldsymbol{x}=\left(x_{\imath} ; i \in \boldsymbol{Z}\right) \in \mathscr{X}, \Theta \boldsymbol{x} \in \mathfrak{X}$ is defined by

$$
(\Theta \boldsymbol{x})_{\jmath}=x_{\jmath+1}, \quad j \in \boldsymbol{Z} .
$$

This induces a shift transformation on the space of functions on $\mathscr{X}$ by

$$
(\Theta \phi)(\boldsymbol{x})=\phi(\Theta \boldsymbol{x}),
$$


and then on $\mathscr{P}(\mathscr{X})$ by

$$
\int \phi d(\Theta \mu)=\int(\Theta \phi) d \mu .
$$

A probability measure $\mu$ on $\mathscr{X}$ is said to be shift invariant if $\Theta \mu=\mu$, and a shift invariant probability measure $\mu$ on $\mathscr{X}$ is said to be ergodic relative to $\Theta$ if any shift invariant measurable function $\phi$ on $\mathscr{X}(\Theta \phi=\phi)$ is constant $\mu$-a.s.. Next we introduce a shift transformation $\tilde{\Theta}$ on $\boldsymbol{D}[0, T]$. For $\boldsymbol{x}(\cdot) \in \boldsymbol{D}[0, T]$, $(\tilde{\Theta}(\boldsymbol{x}(\cdot)))(\cdot) \in \boldsymbol{D}[0, T]$ is defined by

$$
(\tilde{\boldsymbol{\Theta}}(\boldsymbol{x}(\cdot)))(t)=\Theta(\boldsymbol{x}(t)), \quad t \in[0, T] .
$$

We define the shift invariance and the ergodicity of $P \in \mathscr{P}(\boldsymbol{D}[0, T])$ relative to $\tilde{\Theta}$ in the same way as we have defined those of $\mu \in \mathscr{L}(\mathscr{X})$ relative to $\Theta$.

The following theorem concerning the ergodicity of $P_{\mu^{0}}$ will be used for proving a finite cluster property of our process stated in Theorem 1.4 below.

THEOREM 1.3. Suppose that there is a reference measure $\nu$ satisfying (A.1) and (A.3). Let $\mu^{0} \in \mathscr{P}(\mathscr{X})$ satisfy (A.4) and (A.5) and be ergodic relative to $\Theta$. Then $P_{\mu 0}$ is ergodic relative to $\tilde{\Theta}$. When $\mu^{0}=\nu^{\otimes \infty}$ (the equilibrium case), the same result holds under the assumption that $\nu$ satisfies (A.1) and (A.2).

Examples of $\mu^{0} \in \mathscr{L}(\mathscr{X})$ satisfying the assumptions in Theorem 1.3 will be given in Section 4 (see Remark 4.1).

The result concerning a finite cluster property of our process is as follows.

THEOREM 1.4. Suppose that there is a reference measure $\nu$ satisfying (A.1) and (A.3). Let $\mu^{0} \in \mathscr{P}(\mathfrak{X})$ satisfy (A.4) and (A.5) and be ergodic relative to $\Theta$. Then for any $T>0 \quad\left\{\boldsymbol{x}(t), 0 \leqq t \leqq T, P_{\mu 0}\right\}$ has a finite cluster property in the sense that

$$
P_{\mu 0}\left\{\text { the number of } i \geqq 0 \text { satısfying } T_{\imath}=T_{\imath}^{\prime}=T_{\imath+1}^{\prime}=\emptyset \text { is } \infty\right\}=1 \text {, }
$$

$$
P_{\mu 0}\left\{\text { the number of } i<0 \text { satisfying } T_{\imath}=T_{\imath}^{\prime}=T_{\imath+1}^{\prime}=\emptyset \text { is } \infty\right\}=1 \text {, }
$$

where

$$
\begin{aligned}
& T_{\imath}=\left\{0<t \leqq T: x_{i}(t-) \neq x_{i}(t), x_{\imath+1}(t-) \neq x_{\imath+1}(t)\right\}, \\
& T_{\imath}^{\prime}=\left\{0<t \leqq T: x_{i}(t-) \neq x_{i}(t), x_{\imath-1}(t-)=x_{\imath-1}(t), x_{\imath+1}(t-)=x_{\imath+1}(t)\right\} .
\end{aligned}
$$

When $\mu^{0}=\nu^{\otimes \infty}$ (the equilibrium case), the same result holds under the assumption that $\nu$ satisfies (A.1) and (A.2).

In Theorem 1.4 we formulated the finite cluster property of our process on the path space $\left(\boldsymbol{D}[0, T], P_{\mu 0}\right)$. We now give some remarks concerning the statement of it. We first consider the case where 


$$
\pi\left(x, y ;\left\{\left(x, y^{\prime}\right): y^{\prime} \neq y\right\}\right)=\pi\left(x, y ;\left\{\left(x^{\prime}, y\right): x^{\prime} \neq x\right\}\right)=0 \text { for all } x, y \in \boldsymbol{R} .
$$

Then the change of the value of the $i$-th spin of the process $\{\boldsymbol{X}(\cdot)\}$ at time $t$, namely, the event " $X_{i}(t-) \neq X_{i}(t)$ " occurs either with $X_{\imath+1}(t-) \neq X_{\imath+1}(t)$ or with $X_{\imath-1}(t-) \neq X_{\imath-1}(t)$; the former is due to $(i, i+1)$-interaction, the latter is due to $(i-1, i)$-interaction and these two interactions do not occur at the same time. Thus the change $X_{i}(t-) \neq X_{i}(t)$ is a consequence of $(i, i+1)$-interaction only if $X_{\imath+1}(t-) \neq X_{\imath+1}(t)$. In this case the assertions (1.7) and (1.8) in Theorem 1.4 may be replaced, respectively, by

$$
\begin{aligned}
& P_{\mu 0}\left\{\text { the number of } i \geqq 0 \text { satisfying } T_{\imath}=\emptyset \text { is } \infty\right\}=1, \\
& \left.P_{\mu 0} \text { the number of } i<0 \text { satisfying } T_{\imath}=\emptyset \text { is } \infty\right\}=1 .
\end{aligned}
$$

When the condition (1.9) is not satisfied, the change of the value of the $i$-th spin at time $t$ due to $(i, i+1)$-interaction takes place in the following two ways:

$$
\begin{aligned}
& \text { (i) } X_{i}(t-) \neq X_{i}(t), X_{\imath+1}(t-) \neq X_{\imath+1}(t) . \\
& \text { (ii) } X_{i}(t-) \neq X_{i}(t), X_{\imath-1}(t-)=X_{\imath-1}(t), X_{\imath+1}(t-)=X_{\imath+1}(t) .
\end{aligned}
$$

Note that (ii) contains a result of $(i-1, i)$-interaction as well as $(i, i+1)$-interaction. To state the finite cluster property in Theorem 1.4, the above two ways of the change are taken into account.

In Section 2 we give some remarks concerning finite systems such as $\boldsymbol{X}^{M}(t)$, $t \geqq 0$. We prove Theorem 1.1 and Theorem 1.2 in Section 3, and prove Theorem 1.3 and Theorem 1.4 in Section 4.

In the rest of this section, we give some examples of $\pi(x, y ; \cdot)$ and $c(x, y)$ for which there exists a reference measure $\nu$ satisfying (A.1).

Example 1.1. For $x, y \in \boldsymbol{R}$, let $\pi(x, y ; \cdot)=\boldsymbol{\delta}_{(y, x)}(\cdot)$, where $\boldsymbol{\delta}_{(x, y)}$ is the $\delta$-measure at $(x, y) \in \boldsymbol{R}^{2}$, and assume $c(x, y)=c(y, x)$. Then (A.1) is satisfied for all $\nu \in \mathscr{Q}(\boldsymbol{R})$. Moreover the corresponding Markov process on $\boldsymbol{R}^{2}$ is reversible relative to $\nu \otimes \nu$ for all $\nu \in \mathscr{Q}(\boldsymbol{R})$. The Markov process on $\mathscr{X}$ obtained in Theorem 1.1 or Theorem 1.2 in this case is called an exchange process. In the case where $x, y \in\{0,1\}$, it is a well-known exclusion process.

Example 1.2. The dynamics of Kac's caricature of a Maxwellian gas described below satisfies our assumption. In this model a real number $x$ is viewed as a velocity. Let $(x, y)$ be a pair of velocities. At random times the pair $(x, y)$ is transformed into

$$
\left(x^{\prime}, y^{\prime}\right)=(x \cos \theta-y \sin \theta, x \sin \theta+y \cos \theta),
$$

where $\theta$ is distributed according to $Q \in \mathscr{P}([-\pi, \pi])$. In this transformation the sum of energy $x^{2}+y^{2}$ is conserved. If we take $\pi(x, y ; \cdot)$ as the law of $\left(x^{\prime}, y^{\prime}\right)$ under $Q$ and $c(x, y)=c\left(x^{2}+y^{2}\right)$ for a function $c(\cdot)$ on $[0, \infty)$, then (A.1) is 
satisfied for $\nu=N(0, v)$, the normal distribution with expectation 0 and variance $v>0$. In particular, if we assume that $Q$ is symmetric, then the corresponding Markov process on $\boldsymbol{R}^{2}$ is reversible relative to $\nu \otimes \nu$ for $\nu=N(0, v)$.

Example 1.3. Let $X$ and $Y$ be independent $\boldsymbol{R}$-valued random variables with a common distribution $\nu \in \mathscr{P}(\boldsymbol{R})$, and $\pi(x, y ; \cdot), x, y \in \boldsymbol{R}$, be the conditional distribution of $(X, Y)$ given $c(X, Y)=c(x, y)$, where $c(x, y)$ is a given function on $\boldsymbol{R}^{2}$. Then (A.1) is satisfied for the common distribution $\nu$. Moreover the corresponding Markov process on $R^{2}$ is reversible relative to $\nu \otimes \nu$. In the case where $\nu \in \mathscr{L}((0, \infty))$ and $c(x, y)=x+y, x, y>0$, the hydrodynamic limit is studied for the corresponding Markov process on a periodic lattice in [4] and [5], and for the Markov process on $\mathscr{X}$ obtained in Theorem 1.2 in [6] under some additional assumptions for $\nu$ and an initial distribution. This dynamics is an extension of that of the pair of energy $\left(x^{2}, y^{2}\right)$ in Example 1.2 when $Q(d \theta)=d \theta$. In Example 1.2 the distribution of energy is $\nu(d x)=(1 / \sqrt{ } 2 \pi v x) e^{-x /(2 v)} d x, x>0$, $v>0$.

\section{Some preliminaries for finite systems}

In this section we give some preliminary observations concerning Markov processes of finite systems. The lemmas of this section are mostly known; they are stated because of their importance for the proof of our main theorems.

Let $\Lambda$ be a finite subset of $\boldsymbol{Z}$ and consider a Markov process

$$
\left\{\boldsymbol{X}^{(\Lambda)}\right\}=\left\{\boldsymbol{X}^{(\Lambda)}(t), t \geqq 0\right\}=\left\{\left(X_{i}^{(\Lambda)}(t) ; i \in \boldsymbol{Z}\right), t \geqq 0\right\}
$$

on $\mathscr{X}$ with generator $\mathcal{L}^{(\Lambda)}=\sum_{i \in \Lambda} L_{\imath}$. For the process $\left\{\boldsymbol{X}^{(\Lambda)}\right\}$ the values of spins on those sites $i$ 's for which $i \notin \Lambda$ and $i-1 \notin \Lambda$ remain unchanged with time. Thus the process $\left\{\boldsymbol{X}^{(\Lambda)}\right\}$ is regarded as a process on

$$
\mathscr{X}^{(\Lambda)}=\boldsymbol{R}^{\Lambda \cup(\Lambda+1)}=\left\{\boldsymbol{x}_{\Lambda}=\left(x_{\imath} ; i \in \Lambda \cup(\Lambda+1)\right): x_{i} \in \boldsymbol{R} \text { for } i \in \Lambda \cup(\Lambda+1)\right\},
$$

where $\Lambda+1=\{i \in \boldsymbol{Z}: i-1 \in \Lambda\}$. For $\phi=\phi\left(\boldsymbol{x}_{\Lambda}\right), \boldsymbol{x}_{\Lambda} \in \mathfrak{X}^{(\Lambda)}, \mathcal{L}^{(\Lambda)} \phi$ can be expressed as

$$
\mathcal{L}^{(\Lambda)} \phi\left(\boldsymbol{x}_{\Lambda}\right)=q^{(\Lambda)}\left(\boldsymbol{x}_{\Lambda}\right)\left\{\int \pi^{(\Lambda)}\left(\boldsymbol{x}_{\Lambda}, d \boldsymbol{y}_{\Lambda}\right) \phi\left(\boldsymbol{y}_{\Lambda}\right)-\phi\left(\boldsymbol{x}_{\Lambda}\right)\right\},
$$

where

$$
\begin{aligned}
& q^{(\Lambda)}\left(\boldsymbol{x}_{\Lambda}\right)=\sum_{\imath \in \Lambda} c\left(x_{\imath}, x_{\imath+1}\right)+1 \\
& \pi^{(\Lambda)}\left(\boldsymbol{x}_{\Lambda}, d \boldsymbol{y}_{\Lambda}\right) \\
& =\frac{1}{q^{(\Lambda)}\left(\boldsymbol{x}_{\Lambda}\right)}\left\{\sum_{i \in \Lambda} c\left(x_{\imath}, x_{\imath+1}\right) \pi\left(x_{\imath}, x_{\imath+1} ; d y_{i} d y_{\imath+1}\right) \prod_{j \neq \imath, \imath+1} \delta_{x_{j}}\left(d y_{j}\right)+\delta_{x_{\Lambda}}\left(d \boldsymbol{y}_{\Lambda}\right)\right\} .
\end{aligned}
$$


The function $q^{(\Lambda)}\left(\boldsymbol{x}_{A}\right)$ is strictly positive and $\pi^{(\Lambda)}\left(\boldsymbol{x}_{A}, d \boldsymbol{y}_{A}\right)$ is a transition probability on $\mathscr{X}^{(\Lambda)}$. Since $q^{(\Lambda)}\left(\boldsymbol{x}_{A}\right)$ is unbounded in general, it is not obvious that the process $\left\{\boldsymbol{X}^{(\Lambda)}\right\}$ is conservative, namely, that there is no explosion within a finite time. In the following lemma $\left\{\boldsymbol{X}^{(\Lambda)}\right\}$ is regarded as a process on $\mathfrak{X}^{(\Lambda)}$ and $\nu^{(\Lambda)}\left(d \boldsymbol{x}_{A}\right)$ denotes the product measure $\Pi_{i \in \Lambda \cup(\Lambda+1)} \nu\left(d x_{\imath}\right)$.

LEMMA 2.1. Under the assumption of the existence of a reference measure $\nu$ satisfying (A.1) and

$$
\iint c(x, y) \nu(d x) \nu(d y)<\infty,
$$

the process $\left\{\boldsymbol{X}^{(\Lambda)}\right\}$ is conservative for almost all initial values with respect to $\nu^{(1)}$. Moreover this process has an invariant measure $\nu^{(\Lambda)}$.

Proof. By (A.1) and (A.2'), it follows that

$$
\int \nu^{(\Lambda)}\left(d \boldsymbol{x}_{\Lambda}\right) q^{(\Lambda)}\left(\boldsymbol{x}_{\Lambda}\right) \pi^{(\Lambda)}\left(\boldsymbol{x}_{\Lambda}, B\right)=\int_{B} q^{(\Lambda)}\left(\boldsymbol{x}_{\Lambda}\right) \nu^{(1)}\left(d \boldsymbol{x}_{\Lambda}\right) \text { for all } B \in \mathscr{B}\left(\mathscr{X}^{(\Lambda)}\right)
$$

and

$$
c_{\Lambda} \equiv \int q^{(\Lambda)}\left(\boldsymbol{x}_{\Lambda}\right)^{(\Lambda)}\left(d \boldsymbol{x}_{\Lambda}\right)<\infty .
$$

Define a probability measure $\mu^{(\Lambda)}$ on $\mathscr{X}^{(\Lambda)}$ by

$$
\mu^{(\Lambda)}\left(d \boldsymbol{x}_{\Lambda}\right)=c_{\Lambda}^{-1} q^{(\Lambda)}\left(\boldsymbol{x}_{\Lambda}\right) \nu^{(\Lambda)}\left(d \boldsymbol{x}_{\Lambda}\right) .
$$

Then, by (2.1), we have

$$
\int \mu^{(\Lambda)}\left(d \boldsymbol{x}_{\Lambda}\right) \pi^{(\Lambda)}\left(\boldsymbol{x}_{\Lambda}, B\right)=\mu^{(\Lambda)}(B) \text { for all } B \in \mathcal{B}\left(\mathfrak{X}^{(\Lambda)}\right) .
$$

Let us construct $\left\{\boldsymbol{X}^{(\Lambda)}\right\}$ with initial distribution $\mu^{(\Lambda)}$. Let $\left\{\boldsymbol{X}_{n}, n=0,1,2, \cdots\right\}$ be the discrete time stationary Markov process on $\mathscr{X}^{(\Lambda)}$ with transition probability $\pi^{(\Lambda)}\left(\boldsymbol{x}_{\Lambda}, d \boldsymbol{y}_{\Lambda}\right)$ whose initial (therefore marginal) distribution is $\mu^{(\Lambda)}$, and let $\left\{e_{n}, n=0,1,2, \cdots\right\}$ be an independent family of exponential random variables with mean 1 which is also independent of $\left\{\boldsymbol{X}_{n}, n=0,1,2, \cdots\right\}$. Then the process $\left\{\boldsymbol{X}^{(\Lambda)}\right\}$ with initial distribution $\mu^{(\Lambda)}$ can be obtained as follows:

$$
\boldsymbol{X}^{(\Lambda)}(t)= \begin{cases}\boldsymbol{X}_{0} & \text { if } 0 \leqq t<e_{0} / q^{(\Lambda)}\left(\boldsymbol{X}_{0}\right), \\ \boldsymbol{X}_{n} & \text { if } \sum_{k=0}^{n-1}\left\{e_{k} / q^{(\Lambda)}\left(\boldsymbol{X}_{k}\right)\right\} \leqq t<\sum_{k=0}^{n}\left\{e_{k} / q^{(\Lambda)}\left(\boldsymbol{X}_{k}\right)\right\}, \quad n \geqq 1 .\end{cases}
$$

Once we prove

$$
\sum_{k=0}^{\infty} \frac{e_{k}}{q^{(\Lambda)}\left(\boldsymbol{X}_{k}\right)}=\infty \quad \text { a.s. }
$$


the process $\left\{\boldsymbol{X}^{(A)}\right\}$ will then be conservative for almost all initial values with respect to $\mu^{(\Lambda)}$. Since $\nu^{(\Lambda)}$ is absolutely continuous with respect to $\mu^{(\Lambda)},(2.4)$ also holds when the initial distribution of $\left\{\boldsymbol{X}_{n}, n=0,1,2, \cdots\right\}$ is $\nu^{(\Lambda)}$ and we see that the process $\left\{\boldsymbol{X}^{(\Lambda)}\right\}$ is conservative for almost all initial values with respect to $\nu^{(1)}$. To prove $(2.4)$, we notice that $\left\{\left(X_{n}, e_{n}\right), n=0,1,2, \cdots\right\}$ is a stationary sequence and that $e_{0} / q^{(A)}\left(\boldsymbol{X}_{0}\right)$ is strictly positive almost surely and integrable. Therefore, by Birkhoff's ergodic theorem, we see that

$$
\lim _{n \rightarrow \infty} \frac{1}{n} \sum_{k=0}^{n-1} \frac{e_{k}}{q^{(\Lambda)}\left(\boldsymbol{X}_{k}\right)}
$$

exists almost surely and is strictly positive. Hence (2.4) follows.

Let us prove that $\left\{\boldsymbol{X}^{(\Lambda)}\right\}$ has an invariant measure $\nu^{(1)}$. In the case of bounded $q^{(\Lambda)}\left(\boldsymbol{x}_{A}\right)$ this follows from (2.1). In the unbounded case we prove this by approximating the process by processes with bounded jump rates. For $N \geqq 1$, put $q_{N}^{(\Lambda)}=q^{(\Lambda)} \wedge N$ and define a probability measure $\nu_{N}^{(\Lambda)}$ on $\mathscr{X}^{(\Lambda)}$ by

$$
\nu_{N}^{(\Lambda)}\left(d \boldsymbol{x}_{\Lambda}\right)=\text { const. } \frac{q^{(\Lambda)}\left(\boldsymbol{x}_{\Lambda}\right)}{q_{N}^{(\Lambda)}\left(\boldsymbol{x}_{\Lambda}\right)} \nu^{(\Lambda)}\left(d \boldsymbol{x}_{\Lambda}\right), \quad \text { const. }=\left(\int \frac{q^{(\Lambda)}\left(\boldsymbol{x}_{\Lambda}\right)}{q_{N}^{(\Lambda)}\left(\boldsymbol{x}_{\Lambda}\right)} \nu^{(\Lambda)}\left(d \boldsymbol{x}_{\Lambda}\right)\right)^{-1} .
$$

Using (2.1), we see that $\nu_{N}^{(1)}$ is an invariant measure for the process with jump rate $q_{N}^{(\Lambda)}$ instead of $q^{(\Lambda)}$. We denote this process by $\left\{\boldsymbol{X}_{N, x_{A}}^{(\Lambda)}\right\}=\left\{\boldsymbol{X}_{N, x_{A}}^{(\Lambda)}(t), t \geqq 0\right\}$ when its initial value is $\boldsymbol{x}_{\Lambda} \in \mathscr{X}^{(\Lambda)}$. Let $\boldsymbol{B}\left(\mathscr{X}^{(\Lambda)}\right)$ be the set of bounded Borel functions on $\mathscr{X}^{(\Lambda)}$. From above we have for all $N \geqq 1, t>0$ and $\phi \in \boldsymbol{B}\left(\mathscr{X}^{(\Lambda)}\right)$

$$
\int \nu^{(\Lambda)}\left(d \boldsymbol{x}_{\Lambda}\right) \frac{q^{(\Lambda)}\left(\boldsymbol{x}_{\Lambda}\right)}{q_{N}^{(\Lambda)}\left(\boldsymbol{x}_{\Lambda}\right)} \boldsymbol{E}\left[\phi\left(\boldsymbol{X}_{N, x_{A}}^{(\Lambda)}(t)\right)\right]=\int \phi\left(\boldsymbol{x}_{\Lambda}\right) \frac{q^{(\Lambda)}\left(\boldsymbol{x}_{\Lambda}\right)}{q_{N}^{(\Lambda)}\left(\boldsymbol{x}_{\Lambda}\right)} \nu^{(\Lambda)}\left(d \boldsymbol{x}_{\Lambda}\right) .
$$

We now prove

$$
\int \nu^{(\Lambda)}\left(d \boldsymbol{x}_{\Lambda}\right) \boldsymbol{E}\left[\phi\left(\boldsymbol{X}_{\boldsymbol{x}_{\Lambda}}^{(\Lambda)}(t)\right)\right]=\int \phi\left(\boldsymbol{x}_{\Lambda}\right) \nu^{(\Lambda)}\left(d \boldsymbol{x}_{\Lambda}\right), \quad \phi \in \boldsymbol{B}\left(\mathfrak{X}^{(\Lambda)}\right), \quad t>0,
$$

by taking the limit as $N \rightarrow \infty$ in (2.5). Let $\left\{\boldsymbol{X}_{n}, n=0,1,2, \cdots\right\}$ be the Markov process on $\mathscr{X}^{(\Lambda)}$ with transition probability $\pi^{(\Lambda)}\left(\boldsymbol{x}_{\Lambda}, d \boldsymbol{y}_{\Lambda}\right)$ whose initial distribution is $\nu^{(\Lambda)}$. The processes $\left\{\boldsymbol{X}^{(\Lambda)}\right\}$ and $\left\{\boldsymbol{X}_{N}^{(\Lambda)}\right\}, N \geqq 1$, starting from the same initial value $\boldsymbol{X}_{0}$ are constructed in the same way as in (2.3). For any $t>0$, $n \geqq 0$ and $N \geqq 1$, we observe

$$
\left\{\sum_{k=0}^{n} \frac{e_{k}}{q^{(\Lambda)}\left(\boldsymbol{X}_{k}\right)}>t\right\} \cap\left\{\max _{0 \leqq k \leqq n} q^{(\Lambda)}\left(\boldsymbol{X}_{k}\right)<N\right\} \subset\left\{\boldsymbol{X}_{N}^{(\Lambda)}(s)=\boldsymbol{X}^{(\Lambda)}(s), 0 \leqq s \leqq t\right\} .
$$

Therefore, by (2.4), we get for almost all $\boldsymbol{x}_{\Lambda}$ with respect to $\nu^{(\Lambda)}$

$$
\lim _{N \rightarrow \infty} \boldsymbol{P}\left\{\boldsymbol{X}_{N, x_{A}}^{(\Lambda)}(s)=\boldsymbol{X}_{\boldsymbol{x}_{\Lambda}}^{(\Lambda)}(s), 0 \leqq s \leqq t\right\}=1 .
$$

Notice that for all $N \geqq 1$ and $\boldsymbol{x}_{\Lambda} \in \mathscr{X}^{(\Lambda)}$

$$
\frac{q^{(\Lambda)}\left(\boldsymbol{x}_{\Lambda}\right)}{q_{N}^{(\Lambda)}\left(\boldsymbol{x}_{\Lambda}\right)} \leqq q^{(\Lambda)}\left(\boldsymbol{x}_{\Lambda}\right),
$$


and then let $N \rightarrow \infty$ in (2.5) Then the dominated convergence theorem is applicable by virtue of (2.2) and we obtain (2.6).

For the proof of our main theorems we adopt a special construction of $\left\{\boldsymbol{X}^{(\Lambda)}\right\}$; namely, we construct $\left\{\boldsymbol{X}^{(\Lambda)}\right\}$ by solving the SDE

$$
\begin{aligned}
X_{i}^{(\Lambda)}(t)= & X_{i}+\mathbf{1}_{\Lambda}(i) \int_{0}^{t} \int_{0}^{c\left(X_{i}^{(\Lambda)}(s-), X_{i+1}^{(\Lambda)}(s-)\right)} \int_{0}^{1} \tilde{a} N_{i}(d s d \eta d \xi) \\
& +\mathbf{1}_{\Lambda}(i-1) \int_{0}^{t} \int_{0}^{c\left(X_{i-1}^{(\Lambda)}(s-), X_{i}^{(\Lambda)}(s-)\right)} \int_{0}^{1} \tilde{b} N_{\imath-1}(d s d \eta d \xi), \quad i \in \boldsymbol{Z}, t \geqq 0,
\end{aligned}
$$

where

$$
\begin{aligned}
& \tilde{a}=a\left(\xi ; X_{i}^{(\Lambda)}(s-), X_{i+1}^{(\Lambda)}(s-)\right)-X_{i}^{(\Lambda)}(s-), \\
& \tilde{b}=b\left(\xi ; X_{i-1}^{(\Lambda)}(s-), X_{i}^{(\Lambda)}(s-)\right)-X_{i}^{(\Lambda)}(s-) .
\end{aligned}
$$

In considering the above $\operatorname{SDE}\left\{\boldsymbol{X}^{(\Lambda)}\right\}$ is regarded as a process on $\mathscr{X}$. We assume that the probability law $\mu^{0}$ of the initial value $\boldsymbol{X}=\left(X_{2} ; i \in \boldsymbol{Z}\right)$ satisfies (A.4). Since the projection of $\mu^{0}$ to $\mathscr{X}^{(1)}$ is absolutely continuous with respect to $\prod_{i \in A \cup\left(A_{1}\right)} \nu\left(d x_{2}\right)$, there exists a unique solution of (2.7) by Lemma 2.1 .

Let $M=\left\{M^{-}, M^{+}\right\}$be a pair of integers $M^{-}$and $M^{+}$such that $M^{-} \leqq-1$ and $M^{+} \geqq 1$ and let $i$ and $j$ be fixed integers satisfying $M^{-} \leqq i<j \leqq M^{+}-1$. In the course of the proof of our main theorems, what we need is the finite system $\left\{\boldsymbol{X}^{(\Lambda)}\right\}$ in which $\Lambda$ takes the form $\left[M^{-}, M^{+}\right),\left[M^{-}, M^{+}\right)-\{i\}$ or $\left[M^{-}, M^{+}\right)-\{\imath, j\}$. For simplicity we denote the process $\left\{\boldsymbol{X}^{(\Lambda)}\right\}$ for the first form of $\Lambda$ by $\left\{X^{M}\right\}$, for the second by $\left\{X^{M, i}\right\}$ and for the third by $\left\{X^{M, \imath, j}\right\}$. In the process $\left\{X^{M, i}\right\}$ the values of the $i$-th and the $(i+1)$-th spins do not evolve simultaneously. In the process $\left\{X^{M, 2, j}\right\}$ the values of the $i$-th and the $(i+1)$-th spins do not evolve simultaneously and the values of the $j$-th and the $(\jmath+1)$-th spins do not, either. It is also important that the processes $\left\{\boldsymbol{X}^{M}\right\}$, $\left\{\boldsymbol{X}^{M, i}\right\}$ and $\left\{\boldsymbol{X}^{M, \imath, j}\right\}$ are constructed from the same initial value $\boldsymbol{X}$ by solving the SDE's associated with the common Poisson random measures $N_{\imath}, i \in \boldsymbol{Z}$. The validity of Lemma 2.2 which we state later is due to this special construction of our finite systems.

The construction of $\left\{\boldsymbol{X}^{(1)}\right\}$ (solving the SDE (2.7)) is easy; however, we sketch it in order to clarify Lemma 2.2. We put $t_{0}^{(\Lambda)}=0$ and

$$
X_{i}^{(\Lambda)}\left(t_{0}^{(\Lambda)}\right)=X_{i}^{(\Lambda)}(0)=X_{\imath}, \quad i \in \boldsymbol{Z} .
$$

As the first step we define the first interaction time $t_{1}^{(\Lambda)}$ by

where

$$
t_{1}^{(\Lambda)}=\min \left\{t_{1,2}^{(\Lambda)}: i \in \Lambda\right\}
$$

$$
t_{1, i}^{(\Lambda)}=\min \left\{t>0: N_{i}\left(\{t\} \times\left(0, c\left(X_{\imath}, X_{\imath+1}\right)\right] \times(0,1]\right)=1\right\} .
$$

Then there exists a unique index $i(1 ; \Lambda) \in \Lambda$ and $\xi_{1}^{(\Lambda)} \in(0,1]$ such that $t_{1}^{(\Lambda)}=$ $t_{1, i(1 ; \Lambda)}^{(A)}$ and 


$$
N_{i(1 ; A)}\left(\left\{t_{1}^{(\Lambda)}\right\} \times\left(0, c\left(X_{i(1 ; A)}, X_{i(1 ; A)+1}\right)\right] \times\left\{\xi_{1}^{(\Lambda)}\right\}\right)=1 .
$$

We define $X_{i}^{(\Lambda)}\left(t_{1}^{(\Lambda)}\right), i \in \boldsymbol{Z}$, by

$$
X_{i}^{(\Lambda)}\left(t_{1}^{(\Lambda)}\right)= \begin{cases}a\left(\xi_{1}^{(\Lambda)} ; X_{i(1 ; \Lambda)}, X_{i(1 ; \Lambda)+1}\right) & \text { for } i=i(1 ; \Lambda), \\ b\left(\xi_{1}^{(\Lambda)} ; X_{i(1 ; \Lambda)}, X_{i(1 ; \Lambda)+1}\right) & \text { for } i=i(1 ; \Lambda)+1, \\ X_{\imath} & \text { otherwise. }\end{cases}
$$

Suppose the $(n-1)$-th interaction time $t_{n-1}^{(\Lambda)}$ and the corresponding values $X_{i}^{(\Lambda)}\left(t_{n-1}^{(\Lambda)}\right), i \in Z$, are defined $(n \geqq 2)$. We define the $n$-th interaction time $t_{n}^{(\Lambda)}$ by

$$
t_{n}^{(\Lambda)}=\min \left\{t_{n, 2}^{(\Lambda)}: i \in \Lambda\right\}
$$

where

$$
t_{n, i}^{(\Lambda)}=\min \left\{t>t_{n-1}^{(\Lambda)}: N_{i}\left(\{t\} \times\left(0, c\left(X_{i}^{(\Lambda)}\left(t_{n-1}^{(\Lambda)}\right), X_{i+1}^{(\Lambda)}\left(t_{n-1}^{(\Lambda)}\right)\right)\right] \times(0,1]\right)=1\right\} .
$$

Let $i(n ; \Lambda)$ and $\xi_{n}^{(\Lambda)}$ be the unique index in $\Lambda$ and the value in $(0,1]$ such that $t_{n}^{(\Lambda)}=t_{n, i(n ; \Lambda)}^{(\Lambda)}$ and

$$
N_{i(n ; A)}\left(\left\{t_{n}^{(\Lambda)}\right\} \times\left(0, c\left(X_{i(n ; \Lambda)}^{(\Lambda)}\left(t_{n-1}^{(\Lambda)}\right), X_{i(n ; \Lambda)+1}^{(\Lambda)}\left(t_{n-1}^{(A)}\right)\right)\right] \times\left\{\xi_{n}^{(\Lambda)}\right\}\right)=1 .
$$

We then define $X_{i}^{(\Lambda)}\left(t_{n}^{(\Lambda)}\right), i \in Z$, by

$$
X_{i}^{(\Lambda)}\left(t_{n}^{(\Lambda)}\right)= \begin{cases}a\left(\xi_{n}^{(\Lambda)} ; X_{i(n ; \Lambda)}^{(\Lambda)}\left(t_{n-1}^{(\Lambda)}\right), X_{i(n ; \Lambda)+1}^{(\Lambda)}\left(t_{n-1}^{(\Lambda)}\right)\right) & \text { for } i=i(n ; \Lambda), \\ b\left(\xi_{n}^{(\Lambda)} ; X_{i(n ; \Lambda)}^{(\Lambda)}\left(t_{n-1}^{(\Lambda)}\right), X_{i(n ; \Lambda)+1}^{(\Lambda)}\left(t_{n-1}^{(\Lambda)}\right)\right) & \text { for } i=i(n ; \Lambda)+1, \\ X_{i}^{(\Lambda)}\left(t_{n-1}^{(\Lambda)}\right) & \text { otherwise. }\end{cases}
$$

We thus obtain the successive interaction times $t_{n}^{(A)}, n=1,2, \cdots$, and the corresponding values $X_{i}^{(\Lambda)}\left(t_{n}^{(\Lambda)}\right), i \in \boldsymbol{Z}, n=1,2, \cdots$, of the process we want to construct. Lemma 2.1 ensures that $t_{n}^{(\Lambda)} \uparrow \infty$ (a.s.) and our process $\left\{\boldsymbol{X}^{(\Lambda)}\right\}$ can be constructed by

$$
X_{i}^{(\Lambda)}(t)=X_{i}^{(\Lambda)}\left(t_{n}^{(\Lambda)}\right) \quad \text { for } t_{n}^{(\Lambda)} \leqq t<t_{n+1}^{(\Lambda)}, \quad n=0,1, \cdots, i \in Z
$$

Let us examine the first interaction time between the $i$-th and the $(i+1)$-th spins of the process $\left\{\boldsymbol{X}^{M}\right\}$. For any integer $i \in\left[M^{-}, M^{+}\right)$we say that the $i$-th and the $(i+1)$-th spins of the process $\left\{\boldsymbol{X}^{M}\right\}$ interact with each other at time $t$ if

$$
\int_{0}^{t} \int_{0}^{c\left(X_{\imath}^{M}(s-), X_{\imath+1}^{M}(s-)\right)} \int_{0}^{1} N_{i}(d s d \eta d \xi)>\int_{0}^{t-} \int_{0}^{c\left(X_{\imath}^{M}(s-), X_{\imath+1}^{M}(s-)\right)} \int_{0}^{1} N_{i}(d s d \eta d \xi) .
$$

Let $T>0$ and put for any integer $i \in\left[M^{-}, M^{+}\right)$

$$
\tau_{\imath}^{M}=\min T_{\imath}^{M},
$$

where

$T_{i}^{M}=\{0<t \leqq T:$ the $i$-th and the $(i+1)$-th spins of the process $\left\{\boldsymbol{X}^{M}\right\}$ interact with each other at time $\left.t\right\}$. 
Here we adopt the convention that the minimum over the empty set is $\infty$.

LEMMA 2.2. For any $t>0$ and integers $i$ and $j$ satisfying $M^{-} \leqq i<j \leqq M^{+}-1$, the following assertions hold.

$$
\begin{aligned}
&\left\{\tau_{\imath}^{M}>t\right\}=\left\{\int_{0}^{t} \int_{0}^{c\left(X_{\imath}^{M, \imath}(s-), X_{\imath+1}^{M, \imath}(s-)\right)} \int_{0}^{1} N_{i}(d s d \eta d \xi)=0\right\} \\
& \subset\left\{X^{M}(s)=X^{M, \imath}(s) \text { for all } 0 \leqq s \leqq t\right\} \\
&\left\{\tau_{\imath}^{M}>t, \tau_{\jmath}^{M}>t\right\}=\left\{\int_{0}^{t} \int_{0}^{c\left(X_{\imath}^{M, \imath, \jmath}(s-), X_{\imath+1}^{M, \imath, \jmath}(s-)\right)} \int_{0}^{1} N_{i}(d s d \eta d \xi)=0,\right. \\
&\left.\int_{0}^{t} \int_{0}^{c\left(X_{\jmath}^{M, \imath, \jmath}(s-), X_{j+1}^{M, i, j}(s-)\right)} \int_{0}^{1} N_{j}(d s d \eta d \xi)=0\right\} \\
& \subset\left\{X^{M}(s)=X^{M, \imath, \jmath}(s) \text { for all } 0 \leqq s \leqq t\right\}
\end{aligned}
$$

Proof. By the definition of $\tau_{2}^{M}$, we have

$$
\left\{\tau_{\imath}^{M}>t\right\}=\left\{\int_{0}^{t} \int_{0}^{c\left(X_{i}^{M}(s-), X_{i+1}^{M}(s-)\right)} \int_{0}^{1} N_{i}(d s d \eta d \xi)=0\right\} .
$$

Therefore, by the special construction of the processes $\left\{\boldsymbol{X}^{M}\right\}$ and $\left\{\boldsymbol{X}^{M, i}\right\}$ stated above, we obtain (1). We can show (2) in the same way.

\section{Proof of Theorem 1.1 and Theorem 1.2}

In this section we prove Theorem 1.1 and Theorem 1.2. Let $M=\left\{M^{-}, M^{+}\right\}$ be a pair of integers $M^{-}$and $M^{+}$such that $M^{-} \leqq-1$ and $M^{+} \geqq 1$. First we compute the distribution of $\tau_{\imath}^{M}$ and the joint distribution of $\tau_{\imath}^{M}$ and $\tau_{j}^{M}$.

LEMMA 3.1. For any $t>0$ the following assertions hold.

$$
\boldsymbol{P}\left\{\tau_{\imath}^{M}>t\right\}=\boldsymbol{E}\left[\exp \left\{-\int_{0}^{t} c\left(X_{\imath}^{M, \imath}(s), X_{\imath+1}^{M, \imath}(s)\right) d s\right\}\right]
$$

for all $M^{-} \leqq i \leqq M^{+}-1$, where $\boldsymbol{E}$ denotes the expectation with respect to $\boldsymbol{P}$.

$$
\begin{aligned}
& \boldsymbol{P}\left\{\tau_{\imath}^{M}>t, \tau_{\jmath}^{M}>t\right\} \\
& =\boldsymbol{E}\left[\exp \left\{-\int_{0}^{t} c\left(X_{\imath}^{M, \imath, \jmath}(s), X_{\imath+1}^{M, \imath, \jmath}(s)\right) d s-\int_{0}^{t} c\left(X_{\jmath}^{M, \imath, \jmath}(s), X_{\jmath+1}^{M, \imath, \jmath}(s)\right) d s\right\}\right]
\end{aligned}
$$

for all $M^{-} \leqq i<j \leqq M^{+}-1$.

Proof. By Lemma 2.2 (1), we have 


$$
\begin{aligned}
\boldsymbol{P}\left\{\tau_{\imath}^{M}>t\right\}=\boldsymbol{E}\left[\boldsymbol { P } \left\{\int_{0}^{t} \int_{0}^{c\left(X_{\imath}^{M, \imath}(s-), X_{i+1}^{M}{ }^{2}(s-)\right)} \int_{0}^{1} N_{i}(d s d \eta d \xi)=0 \mid\right.\right. \\
\left.\left.N_{k}, M^{-} \leqq k \leqq M^{+}-1, k \neq i\right\}\right] .
\end{aligned}
$$

Note that we have constructed the process $\left\{\boldsymbol{X}^{M, i}\right\}$ without using the Poisson random measure $N_{2}$. Since $N_{k}, k \in Z$, are mutually independent and the intensity measure of $N_{i}(d s d \eta d \xi)$ is $d s d \eta d \xi$, we obtain (1). We can prove (2) in a similar fashion by using Lemma $2.2(2)$.

Let us evaluate the joint distribution of $\tau_{\imath}^{M}$ and $\tau_{\jmath}^{M}$.

LEMMA 3.2. For any $t>0, M^{-} \leqq i<j \leqq M^{+}-1$ and $\beta \geqq 2$,

$$
\begin{aligned}
& \boldsymbol{P}\left\{\tau_{\imath}^{M} \leqq t, \tau_{\jmath}^{M} \leqq t\right\} \\
& \leqq C_{i} C_{j} t^{2-2 / \beta}+\left(C_{i}+C_{j}+C_{\imath, j}+C_{j, \imath}\right)\left(C_{\imath, j}+C_{\jmath, \imath}\right)^{1-1 / \beta} t^{2-3 / \beta+1 / \beta^{2}},
\end{aligned}
$$

where

$$
\begin{aligned}
& C_{\imath}=C_{\imath}^{M, \imath}(t ; \beta)=\left\{\boldsymbol{E}\left[\int_{0}^{t} c\left(X_{\imath}^{M, \imath}(s), X_{\imath+1}^{M, \imath}(s)\right)^{\beta} d s\right]\right\}^{1 / \beta}, \\
& C_{\jmath}=C_{\jmath}^{M, \jmath}(t ; \beta)=\left\{\boldsymbol{E}\left[\int_{0}^{t} c\left(X_{\jmath}^{M, \jmath}(s), X_{\jmath+1}^{M, \jmath}(s)\right)^{\beta} d s\right]\right\}^{1 / \beta}, \\
& C_{\imath, \jmath}=C_{\imath}^{M, \imath, \jmath}(t ; \beta)=\left\{\boldsymbol{E}\left[\int_{0}^{t} c\left(X_{\imath}^{M, \imath, \jmath}(s), X_{\imath+1}^{M, \imath, \jmath}(s)\right)^{\beta} d s\right]\right\}^{1 / \beta}, \\
& C_{\jmath, \imath}=C_{\jmath}^{M, \imath, \jmath}(t ; \beta)=\left\{\boldsymbol{E}\left[\int_{0}^{t} c\left(X_{\jmath}^{M, \imath, \jmath}(s), X_{\jmath+1}^{M, \imath, \jmath}(s)\right)^{\beta} d s\right]\right\}^{1 / \beta} .
\end{aligned}
$$

Proof. For any $t>0$ and $M^{-} \leqq i<\jmath \leqq M^{+}-1$, we have by virtue of Lemma 3.1

where

$$
\boldsymbol{P}\left\{\tau_{\imath}^{M} \leqq t, \tau_{j}^{M} \leqq t\right\}=1-\boldsymbol{E}\left[e^{-a_{\imath}^{M}}\right]-\boldsymbol{E}\left[e^{-a_{j}^{M}}\right]+\boldsymbol{E}\left[e^{-b_{i}^{M}-b_{j}^{M}}\right],
$$

$$
\begin{array}{ll}
a_{\imath}^{M}=\int_{0}^{t} c\left(X_{\imath}^{M, \imath}(s), X_{\imath+1}^{M, \imath}(s)\right) d s, & a_{\jmath}^{M}=\int_{0}^{t} c\left(X_{\jmath}^{M, \jmath}(s), X_{\jmath+1}^{M, \jmath}(s)\right) d s, \\
b_{i}^{M}=\int_{0}^{t} c\left(X_{\imath}^{M, \imath, \jmath}(s), X_{\imath+1}^{M, \imath, \jmath}(s)\right) d s, & b_{j}^{M}=\int_{0}^{t} c\left(X_{\jmath}^{M, \imath, \jmath}(s), X_{\jmath+1}^{M, \imath, \jmath}(s)\right) d s .
\end{array}
$$

Note that for any $s \geqq 0 X_{\imath}^{M, \imath}(s)=X_{\imath}^{M, \imath, \jmath}(s)$ and $X_{j+1}^{M, j}(s)=X_{j+1}^{M, \imath, \jmath}(s)$. By the Lipschitz continuity of $e^{-x}, x \geqq 0$, wee see that

$$
\begin{aligned}
\boldsymbol{P}\left\{\tau_{\imath}^{M} \leqq t, \tau_{j}^{M} \leqq t\right\} & =\boldsymbol{E}\left[\left(1-e^{-a_{\imath}^{M}}\right)\left(1-e^{-a_{j}^{M}}\right)\right]+\boldsymbol{E}\left[e^{-\left(b_{i}^{M}+b_{j}^{M}\right)}-e^{-\left(a_{\imath}^{M}+a_{j}^{M}\right)}\right] \\
& \leqq \boldsymbol{E}\left[a_{\imath}^{M} a_{j}^{M}\right]+\boldsymbol{E}\left[\left|a_{\imath}^{M}-b_{i}^{M}\right|\right]+\boldsymbol{E}\left[\left|a_{j}^{M}-b_{j}^{M}\right|\right] .
\end{aligned}
$$

Applying the Schwarz inequality to the first term of the last line of (3.1), we get 


$$
\begin{aligned}
\boldsymbol{E}\left[a_{\imath}^{M} a_{\jmath}^{M}\right] & \leqq\left\{\boldsymbol{E}\left[\left(a_{\imath}^{M}\right)^{2}\right]\right\}^{1 / 2}\left\{\boldsymbol{E}\left[\left(a_{\jmath}^{M}\right)^{2}\right]\right\}^{1 / 2} \\
& \leqq\left\{\boldsymbol{E}\left[\left(a_{\imath}^{M}\right)^{\beta}\right]\right\}^{1 / \beta}\left\{\boldsymbol{E}\left[\left(a_{\jmath}^{M}\right)^{\beta}\right]\right\}^{1 / \beta}, \quad \beta \geqq 2 .
\end{aligned}
$$

The second inequality in (3.2) follows from the fact that $\left\{\boldsymbol{E}\left[\left(a_{\imath}^{M}\right)^{\beta}\right]\right\}^{1 / \beta}$ is an increasing function of $\beta>0$. As to the second term of the last line of (3.1) we have

$$
\begin{aligned}
& \boldsymbol{E}\left[\left|a_{\imath}^{M}-b_{i}^{M}\right|\right] \\
& =\boldsymbol{E}\left[\left|a_{\imath}^{M}-b_{i}^{M}\right| \mathbf{1}_{\left\{a_{\imath}^{M} \neq b_{i}^{M}\right\}}\right] \\
& \leqq \boldsymbol{E}\left[a_{\imath}^{M} \mathbf{1}_{\left\{a_{\imath}^{M} \neq b_{i}^{M}\right\}}\right]+\boldsymbol{E}\left[b_{i}^{M} \mathbf{1}_{\left\{a_{i}^{M} \neq b_{i}^{M}\right\}}\right] \\
& \leqq\left\{\boldsymbol{E}\left[\left(a_{\imath}^{M}\right)^{\beta}\right]\right\}^{1 / \beta} \boldsymbol{P}\left\{a_{\imath}^{M} \neq b_{i}^{M}\right\}^{1-1 / \beta}+\left\{\boldsymbol{E}\left[\left(b_{i}^{M}\right)^{\beta}\right]\right\}^{1 / \beta} \boldsymbol{P}\left\{a_{\imath}^{M} \neq b_{i}^{M}\right\}^{1-1 / \beta}, \quad \beta>1 .
\end{aligned}
$$

Here we have applied Hölder's inequality. Making use of Hölder's inequality again, we get for $\beta>1$

$$
\left\{\begin{array}{l}
\left(a_{\imath}^{M}\right)^{\beta} \leqq \int_{0}^{t} c\left(X_{\imath}^{M, \imath}(s), X_{\imath+1}^{M, \imath}(s)\right)^{\beta} d s \cdot t^{\beta-1}, \\
\left(b_{i}^{M}\right)^{\beta} \leqq \int_{0}^{t} c\left(X_{i}^{M, \imath, J}(s), X_{\imath+1}^{M, \imath, J}(s)\right)^{\beta} d s \cdot t^{\beta-1} .
\end{array}\right.
$$

Let us estimate $\boldsymbol{P}\left\{a_{\imath}^{M} \neq b_{\imath}^{M}\right\}$. Since for $t<\min \left\{\tau_{\imath}^{M}, \tau_{\jmath}^{M}\right\} \boldsymbol{X}^{M}(s)=\boldsymbol{X}^{M, \imath}(s)=\boldsymbol{X}^{M, \imath, \jmath}(s)$ for all $s \in[0, t]$ by Lemma 2.2, we see that $\left\{a_{\imath}^{M}=b_{i}^{M}\right\} \supset\left\{\tau_{\imath}^{M}>t, \tau_{j}^{M}>t\right\}$. Therefore, by Lemma 3.1, we have

$$
\boldsymbol{P}\left\{a_{i}^{M} \neq b_{i}^{M}\right\} \leqq \boldsymbol{E}\left[1-e^{-\left(b_{i}^{M}+b_{j}^{M}\right)}\right] \leqq \boldsymbol{E}\left[b_{i}^{M}+b_{j}^{M}\right] .
$$

Using Hölder's inequality, we get

$$
\boldsymbol{E}\left[b_{i}^{M}\right] \leqq\left\{\boldsymbol{E}\left[\int_{0}^{t} c\left(X_{\imath}^{M, \imath, \jmath}(s), X_{\imath+1}^{M_{1}, \nu}(s)\right)^{\beta} d s\right]\right\}^{1 / \beta} t^{1-1 / \beta}, \quad \beta>1 .
$$

Combining (3.1) with (3.3)-(3.6), we obtain the required result. The proof of the lemma is finished.

In what follows $K$ denotes a constant that is independent of $i, j$ and $M$ but may vary from place to place.

LEMMA 3.3. (1) Suppose that there is a reference measure $\nu$ satisfying (A.1) and (A.2) and let $\mu^{0}=\nu^{\otimes \infty}$. Then there exists $K>0$ such that

$$
\boldsymbol{P}\left\{\tau_{i}^{M} \leqq t, \tau_{j}^{M} \leqq t\right\} \leqq K\left(t^{2}+t^{3 / 2}\right)
$$

for all $t>0$ and $M^{-} \leqq i<j \leqq M^{+}-1$.

(2) Suppose that there is a reference measure $\nu$ satisfying (A.1) and (A.3) and that $\mu^{0}$ satisfies (A.4). Then there exists $K>0$ such that 


$$
\boldsymbol{P}\left\{\tau_{\imath}^{M} \leqq t, \tau_{j}^{M} \leqq t\right\} \leqq K\left(\left|M^{-}\right| \vee M^{+}\right)^{(2 \alpha) / \beta} t^{2}+K\left(\left|M^{-}\right| \vee M^{+}\right)^{(2 \alpha) / \beta-\alpha / \beta^{2}} t^{2-1 / \beta}
$$

for all $t>0$ and $M^{-} \leqq i<\jmath \leqq M^{+}-1$, where $\alpha$ and $\beta$ are constants in (A.4) and (A.3).

Proof. To show (1), we take $\beta=2$ in Lemma 3.2. Since both $\left\{X^{M, \imath}\right\}$ and $\left\{\boldsymbol{X}^{M, \imath, j}\right\}$ are equilibrium processes with invariant measure $\nu^{\otimes \infty}$, it follows that

$$
\begin{aligned}
\boldsymbol{E}\left[\int_{0}^{t} c\left(X_{\imath}^{M, \imath}(s), X_{\imath+1}^{M, 1}(s)\right)^{2} d s\right] & =\boldsymbol{E}\left[\int_{0}^{t} c\left(X_{\imath}^{M, \imath, \jmath}(s), X_{\imath+1}^{M, \imath, \jmath}(s)\right)^{2} d s\right] \\
& =t \iint c(x, y)^{2} \nu(d x) \nu(d y),
\end{aligned}
$$

which combined with Lemma 3.2 implies the assertion (1).

In proving (2) we estimate $C_{\imath}$ in Lemma 3.2 by making use of the entropy inequality

$$
\int f d \mu \leqq \log \int e^{f} d \nu^{\otimes(2 m+1)}+H_{m}(\mu),
$$

where $f$ is a measurable function on $\boldsymbol{R}^{(-m, \cdots, m\}}$ and $\mu \in \mathscr{P}(\mathscr{X})$. Taking $m=$ $\left|M^{-}\right| \vee M^{+}$and $f\left(\boldsymbol{x}_{m}\right)=\gamma c\left(x_{\imath}, x_{\imath+1}\right)^{\beta}$ in the above where $\gamma$ is a positive constant, we have

$$
\begin{aligned}
& \boldsymbol{E}\left[\int_{0}^{t} c\left(X_{\imath}^{M, \imath}(s), X_{\imath+1}^{M, \imath}(s)\right)^{\beta} d s\right] \\
& \leqq \int_{0}^{t} \frac{1}{\gamma}\left\{\log \int e^{\gamma c(x, y)^{\beta}} \nu(d x) \nu(d y)+H_{\left|M^{-}\right| \vee M^{+}}\left(\mu_{s}^{M, i}\right)\right\} d s .
\end{aligned}
$$

Here $\mu_{s}^{M, \imath} \in \mathscr{Q}(\mathscr{X})$ denotes the distribution of $\boldsymbol{X}^{M, \imath}(s)$. In (3.7) $\left\{\boldsymbol{X}^{M, i}\right\}$ is regarded as a process on $\boldsymbol{R}^{(-m, \cdots, m)}$ with invariant probability measure $\nu^{\otimes(2 m+1)}$ where $m=\left|M^{-}\right| \vee M^{+}$. Therefore $H_{M^{-} \mid \vee M^{+}}\left(\mu_{s}^{M, i}\right)$ is monotone decreasing in $s$ and hence by (A.4)

$$
H_{\left|M^{-}\right| \vee M}+\left(\mu_{s}^{M, i}\right) \leqq C\left(\left|M^{-}\right| \vee M^{+}\right)^{\alpha}
$$

for all $s \geqq 0$. Moreover, by virtue of (A.3) we can take $\beta$ and $\gamma$ in such a way that the first term of the integrand of the right-hand side of (3.7) is finite. Therefore we see that there exists $K>0$ such that

$$
C_{\imath}=C_{\imath}^{M, \imath}(t ; \beta) \leqq K\left(\left|M^{-}\right| \vee M^{+}\right)^{\alpha / \beta} t^{1 / \beta}
$$

for all $t>0$ and $M^{-} \leqq \imath \leqq M^{+}-1$, where $\alpha$ and $\beta$ are constants in (A.4) and (A.3). Since $\left\{\boldsymbol{X}^{M, \imath, j}\right\}$ is also regarded as a process on $\boldsymbol{R}^{\{-m, \cdots, m\}}$ with invariant probability measure $\nu^{\otimes(2 m+1)}$ where $m=\left|M^{-}\right| \vee M^{+}$, we can estimate $C_{\imath, \jmath}$ and $C_{\jmath, \imath}$ in the same way as above. Hence we obtain the required result.

Let $M_{k}=\left\{M_{\bar{k}}^{-}, M_{k}^{+}\right\}, k \geqq 1$, satisfy $-1 \geqq M_{1}^{-} \geqq M_{2}^{-} \geqq \cdots$ and $1 \leqq M_{1}^{+} \leqq M_{2}^{+} \leqq \cdots$ Let us compare the values $X_{0}^{M_{k}}(t), 0 \leqq t \leqq T$, and $X_{0}^{M_{k^{\prime}}}(t), 0 \leqq t \leqq T, k^{\prime}>k$. In 
what follows we consider those $k$ 's for which $\left|M_{k}\right| \wedge M_{k}^{+} \geqq 3$. We then put $m_{k}=\left[\left(\left|M_{k}^{-}\right| \wedge M_{k}^{+}\right) / 3\right]$ and $\delta_{k}=T / m_{k}$, where $[u]$ denotes the integral part of $u \in \boldsymbol{R}$. Divide the time interval $[0, T]$ into short pieces of length $\delta_{k}$. For the processes $\left\{\boldsymbol{X}^{M_{k}}\right\}$ and $\left\{\boldsymbol{X}^{M_{k^{\prime}}}\right\}, k^{\prime}>k$, during the time interval $\left((l-1) \boldsymbol{\delta}_{k}, l \boldsymbol{\delta}_{k}\right]$ where $1 \leqq l \leqq m_{k}$, we observe the interactions between spins on each pair of adjacent sites in $\left[3\left(m_{k}-l\right), 3\left(m_{k}-l+1\right)\right]$ or in $\left[-3\left(m_{k}-l+1\right),-3\left(m_{k}-l\right)\right]$. To be precise we introduce the events

$$
\begin{aligned}
& A_{l, 1}^{(k)}=\left\{\#\left\{0 \leqq \jmath \leqq 2: T_{3\left(m_{k}-l\right)+j}^{M_{k}} \cap\left((l-1) \delta_{k}, l \delta_{k}\right]=\emptyset\right\} \geqq 2\right\}, \\
& A_{l, 2}^{(k)}=\left\{\#\left\{0 \leqq j \leqq 2: T_{-3\left(m_{k}-l+1\right)+j}^{M_{k}} \cap\left((l-1) \delta_{k}, l \delta_{k}\right]=\emptyset\right\} \geqq 2\right\},
\end{aligned}
$$

where $1 \leqq l \leqq m_{k} . \quad A_{l, 1}^{(k)}$ is the event that for the process $\left\{\boldsymbol{X}^{M_{k}}\right\}$ there are two or more pairs of spins on adjacent sites in $\left[3\left(m_{k}-l\right), 3\left(m_{k}-l+1\right)\right]$ which do not interact with each other during the interval $\left((l-1) \delta_{k}, l \delta_{k}\right]$. The event $A_{l, 2}^{(k)}$ is related to the interval $\left[-3\left(m_{k}-l+1\right),-3\left(m_{k}-l\right)\right]$ and can be explained in the same way as $A_{l, 1}^{(k)}$. For $k^{\prime}>k$ and $1 \leqq l \leqq m_{k}$, we put

$$
\begin{aligned}
& B_{l, 1}^{\left(k^{\prime}\right)}=\left\{\#\left\{0 \leqq j \leqq 2: T_{3\left(m_{k}-l\right)+j}^{M_{k^{\prime}}} \cap\left((l-1) \delta_{k}, l \delta_{k}\right]=\emptyset\right\} \geqq 2\right\}, \\
& B_{l, 2}^{\left(k^{\prime}\right)}=\left\{\#\left\{0 \leqq \jmath \leqq 2: T_{-3\left(m_{k}-l+1\right)+j}^{M_{k^{\prime}}} \cap\left((l-1) \delta_{k}, l \delta_{k}\right]=\emptyset\right\} \geqq 2\right\} .
\end{aligned}
$$

These events for the process $\left\{\boldsymbol{X}^{M_{k^{\prime}}}\right\}$ correspond to $A_{l, r}^{(k)}, r=1,2$, for the process $\left\{\boldsymbol{X}^{M_{k}}\right\}$.

LEMMA 3.4. For any $k$ and $k^{\prime}>k$

$$
\bigcap_{l=1}^{m_{k}} \bigcap_{r=1}^{2}\left(A_{l, r}^{(k)} \cap B_{l, r}^{\left(k^{\prime}\right)}\right) \subset\left\{X_{0}^{M} k(t)=X_{0}^{M_{k^{\prime}}}(t) \text { for all } t \in[0, T]\right\} \text {. }
$$

Proof. First consider the events $A_{1,1}^{(k)}$ and $B_{1,1}^{\left(k^{\prime}\right)}$. Rewriting these in terms of $\tau_{\imath}^{M}$ and $\tau_{\imath}^{M k^{\prime}}$, we have

$$
\begin{aligned}
& A_{1,1}^{(k)}=\left\{\#\left\{3 m_{k}-3 \leqq i \leqq 3 m_{k}-1: \tau_{\imath}^{M}{ }_{k}>\delta_{k}\right\} \geqq 2\right\}, \\
& B_{1,1}^{\left(k^{\prime}\right)}=\left\{\#\left\{3 m_{k}-3 \leqq i \leqq 3 m_{k}-1: \tau_{\imath}^{M_{k^{\prime}}}>\delta_{k}\right\} \geqq 2\right\} .
\end{aligned}
$$

$A_{1,1}^{(k)} \cap B_{1,1}^{\left(k^{\prime}\right)}$ is the event that for each of the processes $\left\{\boldsymbol{X}^{M_{k}}\right\}$ and $\left\{\boldsymbol{X}^{M_{k^{\prime}}}\right\}$ there are two or more pairs of spins on adjacent sites in $\left[3 m_{k}-3,3 m_{k}\right]$ which do not interact with each other up to time $\delta_{k}$. Thus this is included in the event that there exists a pair of adjacent sites in $\left[3 m_{k}-3,3 m_{k}\right]$ such that the corresponding spins do not interact with each other up to time $\delta_{k}$ either for $\left\{\boldsymbol{X}^{M_{k}}\right\}$ or for $\left\{\boldsymbol{X}^{M_{k^{\prime}}}\right\}$, namely, we have

$$
A_{1,1}^{(k)} \cap B_{1,1}^{\left(k^{\prime}\right)} \subset \bigcup_{\imath=3 m_{k}-3}^{3 m_{k}-1}\left\{\tau_{\imath}^{M k}>\delta_{k}, \tau_{\imath}^{M_{k^{\prime}}}>\boldsymbol{\delta}_{k}\right\} .
$$

As to $A_{1,2}^{(k)} \cap B_{1,2}^{\left(k^{\prime}\right)}$, the same argument works concerning the interactions between spins on each pair of adjacent sites in $\left[-3 m_{k},-3 m_{k}+3\right]$ and we have 


$$
A_{1,2}^{(k)} \cap B_{1,2}^{\left(k^{\prime}\right)} \subset \bigcup_{\jmath=-3 m_{k}}^{-3 m_{k}+2}\left\{\tau_{\jmath}^{M}{ }_{k}>\delta_{k}, \tau_{\jmath}^{M_{k^{\prime}}}>\delta_{k}\right\} .
$$

Therefore we get

$$
\begin{aligned}
& \bigcap_{r=1}^{2}\left(A_{1, r}^{(k)} \cap B_{1, r}^{\left(k^{\prime}\right)}\right) \\
& \subset \bigcup_{\imath=3 m_{k}-3}^{3 m_{k}-1} \bigcup_{j=-3 m_{k}}^{-3 m_{k}+2}\left\{\tau_{\imath}^{M m_{k}}>\delta_{k}, \tau_{\jmath}^{M}>\delta_{k}, \tau_{\imath}^{M_{k^{\prime}}}>\delta_{k}, \tau_{\jmath}^{M_{k^{\prime}}}>\delta_{k}\right\} \\
& \subset \bigcup_{\imath=3 m_{k}-3}^{3 m_{k}-1} \bigcup_{\jmath=-3 m_{k}}^{-3 m_{k}+2}\left\{\boldsymbol{X}^{M_{k}}(t)=\boldsymbol{X}^{M_{k}, \jmath, \imath}(t), \boldsymbol{X}^{M_{k^{\prime}}}(t)=\boldsymbol{X}^{M_{k^{\prime}}, \jmath, \imath}(t) \text { for all } 0 \leqq t \leqq \delta_{k}\right\} .
\end{aligned}
$$

The last inclusion relation in (3.8) comes from Lemma $2.2(2)$. Since $X_{n}^{M}{ }_{k},{ }^{, \imath}(t)$ $=X_{n}^{M_{k^{\prime}, j, \imath}}(t)$ for all $t \geqq 0$ and $j+1 \leqq n \leqq i$, the set in the last line of (3.8) is included in

$$
\left\{X_{n}^{M}{ }_{k}(t)=X_{n}^{M^{\prime}}(t) \text { for all }-3 m_{k}+3 \leqq n \leqq 3 m_{k}-3 \text { and } 0 \leqq t \leqq \delta_{k}\right\} .
$$

Next consider the event $\bigcap_{l=1}^{2} \bigcap_{r=1}^{2}\left(A_{l, r}^{(k)} \cap B_{l, r}^{\left(k^{\prime}\right)}\right)$. Since this is included in the set $\left\{X_{n}^{M}\left(\boldsymbol{\delta}_{k}\right)=X_{n}^{M_{k^{\prime}}}\left(\boldsymbol{\delta}_{k}\right)\right.$ for all $\left.-3 m_{k}+3 \leqq n \leqq 3 m_{k}-3\right\}$ by (3.9), the same argument as above leads to

$$
\begin{aligned}
& \bigcap_{l=1}^{2} \bigcap_{r=1}^{2}\left(A_{l, r}^{(k)} \cap B_{l, r}^{\left(k^{\prime}\right)}\right) \\
& \subset\left\{X_{n}^{M}(t)=X_{n}^{M_{k^{\prime}}}(t) \text { for all }-3 m_{k}+6 \leqq n \leqq 3 m_{k}-6 \text { and } 0 \leqq t \leqq 2 \delta_{k}\right\} .
\end{aligned}
$$

Repeating this to $\cap_{l=1}^{m_{k}} \cap_{r=1}^{2}\left(A_{l, r}^{(k)} \cap B_{l, r}^{\left(k^{\prime}\right)}\right)$, we arrive at the required result.

Proof of Theorem 1.1. Let us estimate the probability of the event $\left\{X_{0}^{M} k(t) \neq X_{0}^{M_{k^{\prime}}}(t)\right.$ for some $\left.t \in[0, T]\right\}$ for (large) $k \geqq 1$ and $k^{\prime}>k$. For this, by virtue of Lemma 3.4, we have only to estimate the probability of each of the events $\left(A_{l, r}^{(k)}\right)^{c}$ and $\left(B_{l, r}^{\left(k^{\prime}\right)}\right)^{c}$ for $1 \leqq l \leqq m_{k}, r=1,2 . \quad\left(A_{1,1}^{(k)}\right)^{c}$ is the event that for $\left\{\boldsymbol{X}^{\boldsymbol{M}_{k}}\right\}$ there are two or more pairs of spins on adjacent sites in $\left[3 m_{k}-3,3 m_{k}\right]$ which actually interact with each other up to time $\delta_{k}$. So it follows that

$$
\begin{aligned}
\boldsymbol{P}\left\{\left(A_{1,1}^{(k)}\right)^{c}\right\} \leqq & \boldsymbol{P}\left\{\tau_{3 m_{k}-3}^{M_{k}} \leqq \boldsymbol{\delta}_{k}, \tau_{3 m_{k-2}}^{M_{k}} \leqq \boldsymbol{\delta}_{k}\right\}+\boldsymbol{P}\left\{\tau_{3 m_{k^{-3}}}^{M_{k}} \leqq \boldsymbol{\delta}_{k}, \tau_{3 m_{k^{-1}}}^{M_{k}} \leqq \boldsymbol{\delta}_{k}\right\} \\
& +\boldsymbol{P}\left\{\tau_{3 m_{k}-2}^{M_{k}} \leqq \boldsymbol{\delta}_{k}, \tau_{3 m_{k}-1}^{M_{k}} \leqq \boldsymbol{\delta}_{k}\right\} .
\end{aligned}
$$

Recall $\delta_{k}=T /\left[\left(\left|M_{k}^{-}\right| \wedge M_{k}^{+}\right) / 3\right]$. By (3.10) and Lemma $3.3(1)$, we see that there exists $K>0$ such that

$$
\boldsymbol{P}\left\{\left(A_{1,1}^{(k)}\right)^{c}\right\} \leqq K\left(\left|M_{k}^{-}\right| \wedge M_{k}^{+}\right)^{-3 / 2}
$$

for all $k \geqq 1$ satisfying $\delta_{k}<1$. Concerning the probability of each of the events $\left(A_{1,2}^{(k)}\right)^{c}$ and $\left(B_{1, r}^{\left(k^{\prime}\right)}\right)^{c}, r=1,2$, we have the same estimates as above. Let us consider the events for $l>1$. Since $\left\{\boldsymbol{X}^{M}\right\}$ is an equilibrium process, the distribution of $\boldsymbol{X}^{M}(t)$ is $\nu^{\otimes \infty}$ for $t \geqq 0$. Therefore we can estimate $\boldsymbol{P}\left\{\left(A_{l, r}^{(k)}\right)^{c}\right\}$ and $\boldsymbol{P}\left\{\left(B_{l, r}^{\left(k^{\prime}\right)}\right)^{c}\right\}$ 
by applying Lemma $3.3(1)$ in the case $l>1$, too. Since the estimate therein does not depend on the sites, we obtain the same estimates as in $l=1$. More precisely, there exists $K>0$ such that

$$
\boldsymbol{P}\left\{\left(A_{i, r}^{(k)}\right)^{c}\right\} \leqq K\left(\left|M_{k}^{-}\right| \wedge M_{k}^{+}\right)^{-3 / 2}, \quad \boldsymbol{P}\left\{\left(B_{i, r}^{\left(k^{\prime}\right)}\right)^{c}\right\} \leqq K\left(\left|M_{k}^{-}\right| \wedge M_{k}^{+}\right)^{-3 / 2}
$$

for all $k \geqq 1$ satisfying $\delta_{k}<1, k^{\prime}>k, 1 \leqq l \leqq m_{k}$ and $r=1,2$. Combining this with Lemma 3.4 , we have

$$
\boldsymbol{P}\left\{X_{0}^{M}{ }_{k}(t) \neq X_{0}^{M} k_{k^{\prime}}(t) \text { for some } t \in[0, T]\right\} \leqq K^{\prime}\left(\left|M_{\bar{k}}^{-}\right| \wedge M_{k}^{+}\right)^{-1 / 2}
$$

for some $K^{\prime}>0$ independent of $k$ and $k^{\prime}>k$. If $\left\{M_{k}, k \geqq 1\right\}$ satisfies (A.6), then it follows that

$$
\sum_{k=1}^{\infty} \boldsymbol{P}\left\{X_{0}^{M} k(t) \neq X_{0}^{M} k+1(t) \text { for some } t \in[0, T]\right\}<\infty
$$

Therefore by Borel-Cantelli's lemma

$\boldsymbol{P}$ \{there exists $X_{0}(t)$ such that $X_{0}(t)=X_{0}^{M}(t)$

for all $t \in[0, T]$ and for all sufficiently large $k\}=1$.

Since a similar argument works for any site $i \neq 0$, we obtain (1.4).

Finally let us prove that $\boldsymbol{X}(t)=\left(X_{i}(t) ; i \in \boldsymbol{Z}\right), t \geqq 0$, obtained above does not depend on $\left\{M_{k}, k \geqq 1\right\}$ satisfying (A.6). Take $\tilde{M}_{n}=\left\{\tilde{M}_{n}^{-}, \tilde{M}_{n}^{+}\right\}, n \geqq 1$, satisfying $-1 \geqq \tilde{M}_{1}^{-} \geqq \tilde{M}_{2}^{-} \geqq \cdots, 1 \leqq \tilde{M}_{1}^{+} \leqq \tilde{M}_{2}^{+} \leqq \cdots$ and (A.6). Then

$$
\boldsymbol{P}\left\{\text { for any } i \in \boldsymbol{Z} \text { there exists } \tilde{X}_{i}(t) \text { such that } \tilde{X}_{i}(t)=X_{\imath}^{\tilde{\tilde{n}}} n(t)\right.
$$

$$
\text { for all } t \in[0, T] \text { and for all sufficiently large } n\}=1 \text {. }
$$

For $k \geqq 1$ put

$$
n(k)=\min \left\{n \geqq 1: \tilde{M}_{n}^{-} \leqq M_{\bar{k}}^{-}, M_{k}^{+} \leqq \tilde{M}_{n}^{+}\right\} .
$$

Then, since (3.11) holds for any $M_{k}=\left\{M_{\bar{k}}^{-}, M_{k}^{+}\right\}, k \geqq 1$, satisfying $-1 \geqq M_{1}^{-} \geqq$ $M_{2}^{-} \geqq \cdots$ and $1 \leqq M_{1}^{+} \leqq M_{2}^{+} \leqq \cdots$, we have

$$
\boldsymbol{P}\left\{X_{0}^{M}{ }_{k}(t)=X_{0}^{\tilde{M}} n(k)(t) \text { for all } t \in[0, T] \text { and for all sufficiently large } k\right\}=1 .
$$

Therefore we arrive at the assertion for the site 0 :

$$
\boldsymbol{P}\left\{X_{0}(t)=\tilde{X}_{0}(t) \text { for all } t \in[0, T]\right\}=1 .
$$

Since the same argument works for other sites, we obtain the required result.

Proof of Theorem 1.2. If $\left\{M_{k}, k \geqq 1\right\}$ satisfies (A.7) (i), then we see, by Lemma $3.3(2)$, that there exists $K>0$ such that

$$
\begin{aligned}
& \boldsymbol{P}\left\{\tau_{\imath}^{M_{k}} \leqq \boldsymbol{\delta}_{k}, \tau_{\jmath}^{M_{k}} \leqq \boldsymbol{\delta}_{k}\right\} \leqq K\left(M_{k}^{+}\right)^{-q}, \quad M_{k}^{-} \leqq \imath<\jmath \leqq M_{k}^{+}-1, \\
& \boldsymbol{P}\left\{\tau_{\imath}^{M_{k+1}} \leqq \delta_{k}, \tau_{\jmath}^{M_{k+1}} \leqq \boldsymbol{\delta}_{k}\right\} \leqq K\left(M_{k}^{+}\right)^{-q}, \quad M_{k+1}^{-} \leqq i<\jmath \leqq M_{k+1}^{+}-1,
\end{aligned}
$$


for all sufficiently large $k$ and $q=2+\alpha / \beta^{2}-(2 \alpha) / \beta-1 / \beta$. We notice that $1<q<2$ by virtue of (A.5). Thus, by (3.10) and so on we have

$$
\boldsymbol{P}\left\{\left(A_{1, r}^{(k)}\right)^{c}\right\} \leqq K\left(M_{k}^{+}\right)^{-q}, \quad \boldsymbol{P}\left\{\left(B_{1, r}^{(k+1)}\right)^{c}\right\} \leqq K\left(M_{k}^{+}\right)^{-q}
$$

for some $K>0$ independent of $k$ and $r$. Since $\left\{\boldsymbol{X}^{M}\right\}$ is regarded as a process on $\boldsymbol{R}^{\{-m, \cdots, m\}}$ with invariant probability measure $\nu^{\otimes(2 m+1)}$ where $m=\left|M^{-}\right| \vee M^{+}$, $H_{\mid M-I \vee M+}\left(\mu_{t}^{M}\right)$ is monotone decreasing in $t$. Here $\mu_{t}^{M}$ denotes the distribution of $\boldsymbol{X}^{M}(t)$. So $H_{\left|M^{-}\right| \vee M^{+}}\left(\mu_{t}^{M}\right) \leqq C\left(\left|M^{-}\right| \vee M^{+}\right)^{\alpha}$ for all $t \geqq 0$ by (A.4). Therefore we can apply Lemma 3.3 (2) to evaluate the probability of each of the events $\left(A_{l, r}^{(k)}\right)^{c}$ and $\left(B_{l, r}^{(k+1)}\right)^{c}$ for $l>1$, and then we get the same estimates as in $l=1$. More precisely, there exists $K>0$ such that

$$
\boldsymbol{P}\left\{\left(A_{l, r}^{(k)}\right)^{c}\right\} \leqq K\left(M_{k}^{+}\right)^{-q}, \quad \boldsymbol{P}\left\{\left(B_{l, r}^{(k+1)}\right)^{c}\right\} \leqq K\left(M_{k}^{+}\right)^{-q}
$$

for all sufficiently large $k, 1 \leqq l \leqq m_{k}$ and $r=1,2$. Hence we obtain (1.6) in the same way as in the proof of Theorem 1.1 by using (A.7) (ii).

Finally we prove that $\boldsymbol{X}(t)=\left(X_{i}(t) ; i \in \boldsymbol{Z}\right), t \geqq 0$, does not depend on $\left\{M_{k}, k \geqq 1\right\}$ satisfying (A.7). Take $\tilde{M}_{n}=\left\{\tilde{M}_{n}^{-}, \tilde{M}_{n}^{+}\right\}, \quad n \geqq 1$, satisfying $-1 \geqq \tilde{M}_{1}^{-}$ $\geqq \tilde{M}_{2}^{-} \geqq \cdots, 1 \leqq \tilde{M}_{1}^{+} \leqq \tilde{M}_{2}^{+} \leqq \cdots$ and (A.7), and define $n(k), k \geqq 1$, by (3.12). Then we see that there exists $C>1$ such that $\tilde{M}_{n(k)}^{+} \leqq C M_{k}^{+}$for all sufficiently large $k$ by (A.7) (i). Therefore we have

$$
\boldsymbol{P}\left\{X_{0}^{M} k(t)=X_{0}^{\tilde{M}} n(k)(t) \text { for all } t \in[0, T] \text { and for all sufficiently large } k\right\}=1
$$

in the same way as in the proof of (1.6). Hence we arrive at the assertion for the site 0 as in the proof of Theorem 1.1. Since the same argument works for other sites, we obtain the required result.

Remark 3.1. Examples of $\mu^{0} \in \mathscr{Q}(\mathfrak{X})$ satisfying (A.4) are given below. Let $n \geqq 1$ be fixed and consider

$$
\mu^{0}(d \boldsymbol{x})=\prod_{\imath \in \boldsymbol{Z}} f_{i}\left(x_{\imath n}, \cdots, x_{\imath n+n-1}\right) \nu\left(d x_{\imath n}\right) \cdots \nu\left(d x_{\imath n+n-1}\right),
$$

where each $f_{\imath}$ is a probability density on $\boldsymbol{R}^{n}$ with respect to $\nu^{\otimes n}$. First suppose that there exist $C>0$ and $\alpha>0$ satisfying

$$
\int f_{i}\left(x_{0}, \cdots, x_{n-1}\right) \log f_{i}\left(x_{0}, \cdots, x_{n-1}\right) \nu\left(d x_{0}\right) \cdots \nu\left(d x_{n-1}\right) \leqq C(|i|+1)^{\alpha-1}, \quad i \in \boldsymbol{Z} .
$$

Then $\mu^{0}$ in (3.13) satisfies (A.4) for this $\alpha$. Next in (3.13) suppose that for a finite subset $\Lambda$ of $\boldsymbol{Z}, f_{i} \equiv 1, i \notin \Lambda$, and

$$
\int f_{i}\left(x_{0}, \cdots, x_{n-1}\right) \log f_{i}\left(x_{0}, \cdots, x_{n-1}\right) \nu\left(d x_{0}\right) \cdots \nu\left(d x_{n-1}\right)<\infty, \quad i \in \Lambda .
$$

Then $\mu^{0}$ in (3.13) satisfies (A.4) for $\alpha=0$. 
Remark 3.2. When the jump rate $c(\cdot, \cdot)$ is bounded, our process can be constructed for any initial distribution by our method. Indeed, by the proof of Lemma 3.2 we see that there exists $K>0$ such that

$$
\boldsymbol{P}\left\{\tau_{\imath}^{M} \leqq t, \tau_{j}^{M} \leqq t\right\} \leqq K t^{2}
$$

for any $t>0$ and $M^{-} \leqq i<j \leqq M^{+}-1$. Therefore we see that the same conclusion as Theorem 1.2 holds for any initial distribution $\mu^{0}$ under the condition that $M_{k}=\left\{M_{k}^{-}, M_{k}^{+}\right\}, k \geqq 1$, satisfy $-1 \geqq M_{1}^{-} \geqq M_{2}^{-} \geqq \cdots, 1 \leqq M_{1}^{+} \leqq M_{2}^{+} \leqq \cdots$ and

$$
\sum_{k=1}^{\infty}\left(\left|M_{\bar{k}}^{-}\right| \wedge M_{k}^{+}\right)^{-1}<\infty
$$

On the other hand, in this case we can construct the semigroup of our process easily in the following way. Let $\mathscr{D}_{m}$ be the set of bounded Borel functions on $\mathscr{X}$ which depend only on $x_{\imath},-m \leqq i \leqq m$. Our semigroup, denoted symbolically by $\left\{e^{t \mathcal{L}}\right\}$, is constructed on the closure $\overline{U_{m} \mathscr{D}_{m}}$, which is a Banach space with the supremum norm. In fact $C=\sup _{x, y \in R} c(x, y)<\infty$, then for any $\phi \in \mathscr{D}_{m}$, we have $\mathcal{L} \phi \in \mathscr{D}_{m+1}$ and $\|\mathcal{L} \phi\|_{\infty} \leqq 2 C(2 m+2)\|\phi\|_{\infty}$; in general, for $n=1,2, \cdots$ we have $\mathcal{L}^{n} \phi \in \mathscr{D}_{m+n}$ and

$$
\left\|\mathcal{L}^{n} \phi\right\|_{\infty} \leqq(2 C)^{n}(2 m+2)(2 m+4) \cdots(2 m+2 n)\|\phi\|_{\infty} .
$$

Therefore for all $\phi \in \bigcup_{m} \mathscr{D}_{m}, e^{t \mathcal{L}} \phi=\sum_{n=0}^{\infty}\left(t^{n} \mathcal{L}^{n} \phi\right) / n$ ! converges for $0 \leqq t<1 /(4 C)$. Moreover, we can prove that $\left\|e^{t \mathcal{L}} \phi\right\|_{\infty} \leqq\|\phi\|_{\infty}$ for $\phi \in \cup_{m} \mathscr{D}_{m}$ and $0 \leqq t<1 /(4 C)$ (an approximation by a finite system is used for the proof) and hence $e^{t \perp} \phi$, $0 \leqq t<1 /(4 C)$, can be defined for all $\phi \in \overline{\cup_{m} \mathscr{D}_{m}}$. The time $t$ can also be extended to the whole $[0, \infty)$ using the semigroup property.

\section{Proof of Theorem 1.3 and Theorem 1.4}

In this section we prove Theorem 1.3 and Theorem 1.4.

Proof of Theorem 1.3. If $M_{k}=\left\{-M_{k}^{+}, M_{k}^{+}\right\}, k \geqq 1$, satisfy $1 \leqq M_{1}^{+} \leqq M_{2}^{+} \leqq \cdots$ and (A.7) (or (A.6) in the case where $\mu^{0}=\nu^{\infty \infty}$ ) then, for any $i \in \boldsymbol{Z}, i+M_{k} \equiv$ $\left\{i-M_{k}^{+}, i+M_{k}^{+}\right\}, k \geqq 1$, also satisfy (A.7) (or (A.6) respectively). So, by virtue of Theorem 1.2 (or 1.1), we have

$$
\begin{aligned}
& \boldsymbol{P}\left\{\text { for any } i \in \boldsymbol{Z} X_{i}(t)=X_{\imath}^{i+M_{k}(t)}\right. \\
& \quad \text { for all } t \in[0, T] \text { and for all sufficiently large } k\}=1 .
\end{aligned}
$$

Therefore we see that $P_{\mu 0}$ is shift invariant if $\mu^{0}$ is shift invariant.

To prove the ergodicity of $P_{\mu 0}$ we have only to show that, for any pair of functions $\Phi$ and $\Psi$ on $D[0, T]$ which are square integrable with respect to $P_{\mu 0}$,

$$
\lim _{n \rightarrow \infty} \frac{1}{n} \sum_{l=0}^{n-1}\left(\tilde{\Theta}^{l} \Phi\right) \Psi d P_{\mu 0}=\int \Phi d P_{\mu 0} \int \Psi d P_{\mu^{0}} .
$$


In proving (4.2) it is enough to assume that $\Phi$ and $\Psi$ are of the forms

$$
\Phi(\boldsymbol{x}(\cdot))=\phi\left(x_{i}(\cdot), i \in \Lambda\right), \quad \Psi(\boldsymbol{x}(\cdot))=\phi\left(x_{i}(\cdot), i \in \Lambda\right),
$$

where $\Lambda$ is a finite subset of $\boldsymbol{Z}$ and $\phi, \phi$ are bounded measurable functions defined on the space $\boldsymbol{D}[0, T]^{(\Lambda)}$ of functions on $[0, T]$ with values in $\boldsymbol{R}^{\Lambda}$ which are right continuous and have left limits. By (4.1) we have

$$
\begin{aligned}
\frac{1}{n} \sum_{l=0}^{n-1} \int\left(\tilde{\Theta}^{l} \Phi\right) \Psi d P_{\mu 0} & =\frac{1}{n} \sum_{l=0}^{n-1} \boldsymbol{E}\left[\phi\left(X_{\imath+l}(\cdot), i \in \Lambda\right) \psi\left(X_{i}(\cdot), \imath \in \Lambda\right)\right] \\
& =\frac{1}{n} \sum_{l=0}^{n-1} \boldsymbol{E}\left[\phi\left(X_{\imath+l}^{i+l+M_{k}}(\cdot), i \in \Lambda\right) \phi\left(X_{l}^{i+M_{k}}(\cdot), i \in \Lambda\right)\right]+o(1),
\end{aligned}
$$

where $o(1)$ is a term which tends to 0 uniformly in $n$ as $k \rightarrow \infty$. Therefore

$$
\begin{aligned}
& \lim _{n \rightarrow \infty} \frac{1}{n} \sum_{l=0}^{n-1} \int\left(\tilde{\Theta}^{l} \Phi\right) \Psi d P_{\mu 0} \\
& =\lim _{k \rightarrow \infty} \lim _{n \rightarrow \infty} \frac{1}{n} \sum_{l=0}^{n-1} \boldsymbol{E}\left[\phi\left(X_{\imath+l}^{i+l+M_{k}}(\cdot), i \in \Lambda\right) \psi\left(X_{l}^{i+M_{k}}(\cdot), \imath \in \Lambda\right)\right]
\end{aligned}
$$

(at present we do not know the existence of the above limit but at the final stage of the proof we shall see the existence of the limit of the right-hand side first and consequently of the left-hand side). Since $X_{\imath}^{i+M_{k}}(\cdot)$ is determined by the initial value $\left(X_{r} ; i-M_{k}^{+} \leqq r \leqq \imath+M_{k}^{+}\right)$and the Poisson random measures $N_{r}, i-M_{k}^{+} \leqq r \leqq i+M_{k}^{+}-1$, the right-hand side of (4.3) can be written as

$$
\begin{array}{r}
\lim _{k \rightarrow \infty} \lim _{n \rightarrow \infty} \frac{1}{n} \sum_{l=0}^{n-1} \boldsymbol{E}\left[F\left(\left\{X_{r}, r \in\left(I_{1}^{\Lambda}(k)+l\right)\right\} \times\left\{N_{r}, r \in\left(I_{2}^{\Lambda}(k)+l\right)\right\}\right)\right. \\
\left.\times G\left(\left\{X_{r}, r \in I_{1}^{\Lambda}(k)\right\} \times\left\{N_{r}, r \in I_{2}^{\Lambda}(k)\right\}\right)\right]
\end{array}
$$

with suitable functions $F$ and $G$, where $I_{1}^{\Lambda}(k)=\bigcup_{i \in \Lambda}\left\{\jmath \in Z: i-M_{k}^{+} \leqq j \leqq i+M_{k}^{+}\right\}$, $I_{2}^{\Lambda}(k)=\bigcup_{i \in \Lambda}\left\{\jmath \in \boldsymbol{Z}: i-M_{k}^{+} \leqq \jmath \leqq i+M_{k}^{+}-1\right\}$. Since $\boldsymbol{X}=\left(X_{r} ; r \in \boldsymbol{Z}\right)$ and $\left\{N_{r}, r \in \boldsymbol{Z}\right\}$ are independent, the above expectation can be taken separately, namely, first with respect to $\left\{N_{r}\right\}$ and then $\left\{X_{r}\right\}$. To be precise we put

$$
\begin{aligned}
& f(\boldsymbol{x})=\boldsymbol{E}\left[F\left(\left\{x_{r}, r \in I_{1}^{A}(k)\right\} \times\left\{N_{r}, r \in I_{2}^{A}(k)\right\}\right)\right], \\
& g(\boldsymbol{x})=\boldsymbol{E}\left[G\left(\left\{x_{r}, r \in I_{1}^{A}(k)\right\} \times\left\{N_{r}, r \in I_{2}^{A}(k)\right\}\right)\right], \\
& h_{l}(\boldsymbol{x})=\boldsymbol{E}\left[F\left(\left\{x_{r}, r \in\left(I_{1}^{A}(k)+l\right)\right\} \times\left\{N_{r}, r \in\left(I_{2}^{A}(k)+l\right)\right\}\right)\right. \\
& \left.\quad \times G\left(\left\{x_{r}, r \in I_{1}^{A}(k)\right\} \times\left\{N_{r}, r \in I_{2}^{A}(k)\right\}\right)\right],
\end{aligned}
$$

and notice that $h_{l}(\boldsymbol{x})=\Theta^{l} f(\boldsymbol{x}) \cdot g(\boldsymbol{x})$ holds for all sufficiently large $l$, say, for $l>2 M_{k}^{+}-1+\max \Lambda-\min \Lambda$, and (4.4) is equal to

$$
\begin{aligned}
& \lim _{k \rightarrow \infty} \lim _{n \rightarrow \infty} \frac{1}{n} \sum_{l=0}^{n-1} \int_{\mathscr{X}} h_{l}(\boldsymbol{x}) \mu^{0}(d \boldsymbol{x}) \\
& =\lim _{k \rightarrow \infty} \lim _{n \rightarrow \infty} \frac{1}{n} \sum_{l=0}^{n-1} \int_{X} \Theta^{l} f(\boldsymbol{x}) \cdot g(\boldsymbol{x}) \mu^{0}(d \boldsymbol{x})
\end{aligned}
$$




$$
\begin{aligned}
& \left.=\lim _{k \rightarrow \infty} \int_{X} f(\boldsymbol{x}) \mu^{0}(d \boldsymbol{x}) \int_{X} g(\boldsymbol{x}) \mu^{0}(d \boldsymbol{x}) \quad \text { (by the ergodicity of } \boldsymbol{\mu}^{0}\right) \\
& =\lim _{k \rightarrow \infty} \boldsymbol{E}\left[F\left(\left\{X_{r}, r \in I_{1}^{\Lambda}(k)\right\} \times\left\{N_{r}, r \in I_{2}^{\Lambda}(k)\right\}\right)\right] \\
& \quad \times \boldsymbol{E}\left[G\left(\left\{X_{r}, r \in I_{1}^{\Lambda}(k)\right\} \times\left\{N_{r}, r \in I_{2}^{\Lambda}(k)\right\}\right)\right] \\
& =\lim _{k \rightarrow \infty} \boldsymbol{E}\left[\boldsymbol{\phi}\left(X_{\imath}^{i+M_{k}}(\cdot), i \in \Lambda\right)\right] \boldsymbol{E}\left[\phi\left(X_{\imath}^{i+M_{k}}(\cdot), i \in \Lambda\right)\right] \\
& =\boldsymbol{E}\left[\phi\left(X_{i}(\cdot), i \in \Lambda\right)\right] \boldsymbol{E}\left[\phi\left(X_{i}(\cdot), i \in \Lambda\right)\right],
\end{aligned}
$$

which finally equals the right-hand side of (4.2). This completes the proof of Theorem 1.3.

Proof of Theorem 1.4. Define a subset $A$ of $\boldsymbol{D}[0, T]$ by

$$
A=\left\{T_{0}=T_{0}^{\prime}=T_{1}^{\prime}=\emptyset\right\} .
$$

First we show $P_{\mu^{0}}\{A\}>0$. Since the set in which both of the values of the 0 -th and the first spins remain unchanged up to time $T$ is included in $A$, it follows that

$$
\begin{gathered}
P_{\mu 0}\{A\} \geqq \boldsymbol{P}\left\{X_{0}(t)=X_{0} \text { and } X_{1}(t)=X_{1} \text { for all } 0 \leqq t \leqq T\right\} \\
\geqq \boldsymbol{P}\left\{\int_{0}^{T} \int_{0}^{c\left(X_{-1}(s-), X_{0}(s-)\right)} \int_{0}^{1} N_{-1}(d s d \eta d \xi)=0,\right. \\
\int_{0}^{T} \int_{0}^{c\left(X_{0}(s-), X_{1}(s-)\right)} \int_{0}^{1} N_{0}(d s d \eta d \xi)=0, \\
\left.\int_{0}^{T} \int_{0}^{c\left(X_{1}(s-), X_{2}(s-)\right)} \int_{0}^{1} N_{1}(d s d \eta d \xi)=0\right\} .
\end{gathered}
$$

To compute the probability in the last line of (4.5), let us introduce a Markov process on $\mathscr{X}$ which is associated with $\sum_{i \in Z, \imath \neq-1,0,1} L_{\imath}$. Let $M=\left\{M^{-}, M^{+}\right\}$be a pair of integers $M^{-}$and $M^{+}$such that $M^{-} \leqq-2$ and $M^{+} \geqq 3$ and let $\hat{\boldsymbol{X}}^{M}(t)=$ $\left(\hat{X}_{\imath}^{M}(t) ; i \in \boldsymbol{Z}\right), t \geqq 0$, be the Markov process on $\mathscr{X}$ generated by $\sum_{\imath=M^{-}, \imath \neq-1,0,1}^{M^{+}} L_{\imath}$. This is the process $\left\{\boldsymbol{X}^{(\Lambda)}\right\}$ in which $\Lambda=\left[M^{-}, M^{+}\right)-\{-1,0,1\}$ (see Section 2). Note that we have constructed the processes $\left\{\hat{\boldsymbol{X}}^{M}\right\}$ and $\left\{\boldsymbol{X}^{M}\right\}$ from the same initial value $\boldsymbol{X}$ by solving the SDE's associated with the common Poisson random measures $N_{\imath}, i \in \boldsymbol{Z}$. In the same way as in the proof of Theorem 1.2 (or 1.1 in the case where $\mu^{0}=\nu^{\otimes \infty}$ ), we have for any $M_{k}=\left\{M_{\bar{k}}, M_{k}^{+}\right\}, k \geqq 1$, satisfying $-2 \geqq M_{1}^{-} \geqq M_{2}^{-} \geqq \cdots, 3 \leqq M_{1}^{+} \leqq M_{2}^{+} \leqq \cdots$ and (A.7) (or (A.6))

$$
\boldsymbol{P} \text { \{for any } i \in \boldsymbol{Z} \text { there exists } \hat{X}_{i}(t) \text { such that } \hat{X}_{i}(t)=\hat{X}_{\imath}^{M}{ }_{k}(t)
$$

for all $t \in[0, T]$ and for all sufficiently large $k\}=1$.

Moreover we see that $\hat{\boldsymbol{X}}(t)=\left(\hat{X}_{i}(t) ; i \in \boldsymbol{Z}\right), t \geqq 0$, depends neither on $T$ nor on $\left\{M_{k}, k \geqq 1\right\}$ satisfying (A.7) (or (A.6)). For $\hat{\boldsymbol{X}}(t), t \geqq 0$, the values of spins on 
each pair of adjacent sites in $[-1,2]$ do not evolve simultaneously. In particular, $\hat{X}_{0}(t)=X_{0}$ and $\hat{X}_{1}(t)=X_{1}$ for all $t \geqq 0$. By Theorem 1.2 (or 1.1), the probability in the last line of (4.5) can be expressed in terms of finite systems $\left\{\boldsymbol{X}^{\boldsymbol{M}_{k}}\right\}, k \geqq 1$. Therefore, by a slight modification of Lemma 2.2 and also by (4.6) we see the probability is equal to

$$
\begin{aligned}
& \boldsymbol{P}\left\{\int_{0}^{T} \int_{0}^{c\left(\hat{X}_{-1}\left(s_{-}\right), X_{0}\right)} \int_{0}^{1} N_{-1}(d s d \eta d \xi)=0,\right. \\
& \int_{0}^{T} \int_{0}^{c\left(X_{0}, X_{1}\right)} \int_{0}^{1} N_{0}(d s d \eta d \xi)=0, \\
&\left.\int_{0}^{T} \int_{0}^{c\left(X_{1}, \hat{X}_{2}(s-)\right)} \int_{0}^{1} N_{1}(d s d \eta d \xi)=0\right\} \\
&= \boldsymbol{E}\left[\exp \left\{-\int_{0}^{T} c\left(\hat{X}_{-1}(s), X_{0}\right) d s-c\left(X_{0}, X_{1}\right) T-\int_{0}^{T} c\left(X_{1}, \hat{X}_{2}(s)\right) d s\right\}\right] .
\end{aligned}
$$

The above equality is obtained in the same way as in the proof of Lemma 3.1. Let us show that the expectation in the right-hand side of (4.7) is positive. Indeed this is bounded from below by

$$
\begin{array}{r}
\boldsymbol{E}\left[\exp \left\{-\int_{0}^{T} c\left(\hat{X}_{-1}^{M_{k}}(s), X_{0}\right) d s-c\left(X_{0}, X_{1}\right) T-\int_{0}^{T} c\left(X_{1}, \hat{X}_{2}^{M_{k}}(s)\right) d s\right\}\right. \\
\left.\hat{X}_{-1}(s)=\hat{X}_{-1}^{M_{k}}(s) \text { and } \hat{X}_{2}(s)=\hat{X}_{2}^{M_{k}}(s) \text { for all } 0 \leqq s \leqq T\right] .
\end{array}
$$

Since the probability of the set on which the above expectation is taken is positive for sufficiently large $k$ by (4.6) and $\int_{0}^{T} c\left(\hat{X}_{-1}^{M_{k}}(s), X_{0}\right) d s+c\left(X_{0}, X_{1}\right) T+$ $\int_{0}^{T} c\left(X_{1}, \hat{X}_{2}^{M_{k}}(s)\right) d s<\infty$ with positive probability because of the local boundedness of $c(\cdot, \cdot)$, the expectation in (4.8) is positive, and so is $P_{\mu 0}\{A\}$.

For any $i \in \boldsymbol{Z}$, let us introduce a subset $A_{\imath}$ of $\boldsymbol{D}[0, T]$ :

$$
A_{i}=\left\{T_{\imath}=T_{\imath}^{\prime}=T_{\imath+1}^{\prime}=\emptyset\right\}=\tilde{\Theta}^{-\imath} A .
$$

Since $P_{\mu^{0}}$ is ergodic relative to $\tilde{\Theta}$ by Theorem 1.3 , it follows that

$$
\lim _{n \rightarrow \infty} \frac{1}{n} \sum_{\imath=0}^{n-1} \mathbf{1}_{A_{\imath}}=P_{\mu 0}\{A\} \quad\left(P_{\mu 0}-\text { a.s. }\right) \text {. }
$$

Since $P_{\mu 0}\{A\}>0$ as we have shown above, we get $\sum_{\imath=0}^{\infty} \mathbf{1}_{A_{\imath}}=\infty\left(P_{\mu 0}\right.$-a.s. $)$. In like manner, we also obtain $\sum_{\imath=-\infty}^{-1} \mathbf{1}_{A_{i}}=\infty\left(P_{\mu 0}\right.$-a.s. $)$. These are the desired results.

Remark 4.1. The probability measure $\nu^{\otimes \infty}$ is ergodic relative to $\Theta$. Another example of $\mu^{0} \in \mathscr{Q}(\mathscr{X})$ satisfying the assumptions in Theorem 1.3 or 1.4 is given below. Let $n \geqq 1$ be fixed and consider 


$$
\begin{aligned}
& \mu^{0}(d \boldsymbol{x})=\frac{1}{n} \sum_{l=0}^{n-1} \Theta^{\imath} \tilde{\mu}(d \boldsymbol{x}), \\
& \tilde{\mu}(d \boldsymbol{x})=\prod_{i \in Z} f\left(x_{\imath n}, \cdots, x_{\imath n+n-1}\right) \nu\left(d x_{\imath n}\right) \cdots \nu\left(d x_{\imath n+n-1}\right),
\end{aligned}
$$

where $f$ is a probability density on $\boldsymbol{R}^{n}$ with respect to $\nu^{\otimes n}$. If $\int f \log f d \nu^{\otimes n}<\infty$, then $\mu^{0}$ in (4.9) satisfies (A.4) for $\alpha=1$. Moreover, $\mu^{0}$ in (4.9) is ergodic relative to $\Theta$. (Note that $\mu^{0}$ in (4.9) is not a product measure in general $n \geqq 2$.)

Acknowledgement. The author would like to express her thanks to Professor H. Tanaka for helping her with valuable suggestions and constant encouragement. She would also like to thank Professor Y. Tamura for his encouragement.

\section{REFERENCES}

[1] Holley, R., A class of interaction in an infinite particle system, Adv. Math., 5 (1970), 291-309.

[2] Liggett, T.M., Interacting Particle Systems, Springer-Verlag, 1985.

[3] Spitzer, F., Interaction of Markov processes, Adv. Math., 5 (1970), 246-290.

[4] Suzuki, Y. and Uchiyama, K., Hydrodynamic limit for a spin system on a multidimensional lattice, Probab. Theory Related Fields, 95 (1993), 47-74.

[5] Suzuki, Y. AND Uchiyama, K., Hydrodynamic limit for a $[0, \infty)$-valued spin system, to appear in Adv. Stud. Pure Math.

[6] SUZUKI, Y., Hydrodynamic limit for an infinite spin system on $\boldsymbol{Z}$, to appear in Tokyo J. Math.

Department of Mathematics

FACULTY OF SCIENCE AND TECHNOLOGY

KEIO UNIVERSITY

HIYOSHI, YoKoHAMA 223

JAPAN 